BMC

Genomics

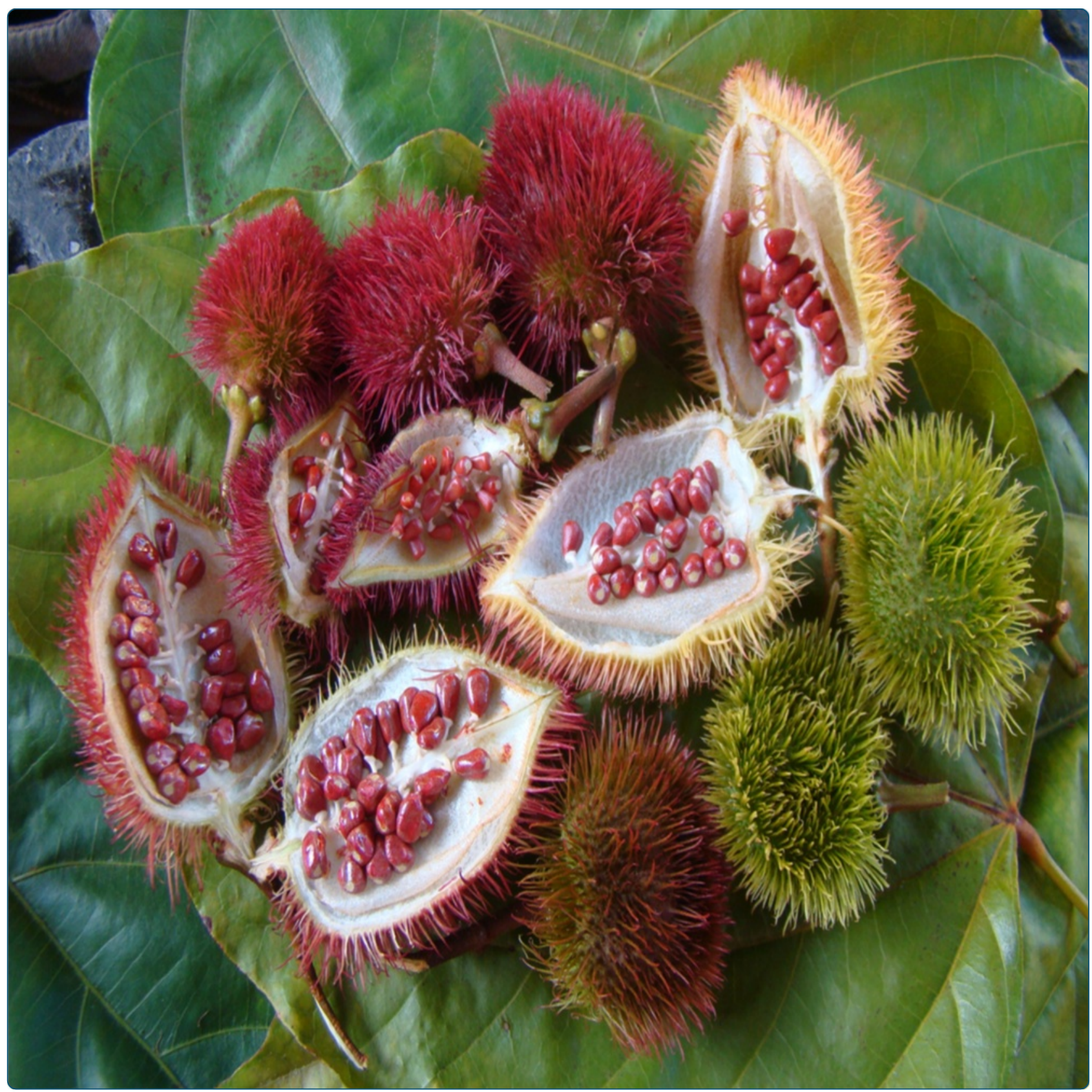

De novo transcriptome sequencing in Bixa orellana to identify genes involved in methylerythritol phosphate, carotenoid and bixin biosynthesis

() Biomed Central 


\title{
De novo transcriptome sequencing in Bixa orellana to identify genes involved in methylerythritol phosphate, carotenoid and bixin biosynthesis
}

Yair Cárdenas-Conejo ${ }^{1}$, Víctor Carballo-Uicab, Meric Lieberman², Margarita Aguilar-Espinosa', Luca Comai² and Renata Rivera-Madrid ${ }^{1 *}$

\begin{abstract}
Background: Bixin or annatto is a commercially important natural orange-red pigment derived from lycopene that is produced and stored in seeds of Bixa orellana L. An enzymatic pathway for bixin biosynthesis was inferred from homology of putative proteins encoded by differentially expressed seed cDNAs. Some activities were later validated in a heterologous system. Nevertheless, much of the pathway remains to be clarified. For example, it is essential to identify the methylerythritol phosphate (MEP) and carotenoid pathways genes.

Results: In order to investigate the MEP, carotenoid, and bixin pathways genes, total RNA from young leaves and two different developmental stages of seeds from B. orellana were used for the construction of indexed mRNA libraries, sequenced on the Illumina HiSeq 2500 platform and assembled de novo using Velvet, CLC Genomics Workbench and CAP3 software. A total of 52,549 contigs were obtained with average length of 1,924 bp. Two phylogenetic analyses of inferred proteins, in one case encoded by thirteen general, single-copy CDNAs, in the other from carotenoid and MEP CDNAs, indicated that B. orellana is closely related to sister Malvales species cacao and cotton. Using homology, we identified 7 and 14 core gene products from the MEP and carotenoid pathways, respectively. Surprisingly, previously defined bixin pathway cDNAs were not present in our transcriptome. Here we propose a new set of gene products involved in bixin pathway.

Conclusion: The identification and qRT-PCR quantification of CDNAs involved in annatto production suggest a hypothetical model for bixin biosynthesis that involve coordinated activation of some MEP, carotenoid and bixin pathway genes. These findings provide a better understanding of the mechanisms regulating these pathways and will facilitate the genetic improvement of B. orellana.
\end{abstract}

Keywords: Annatto, Bixa orellana, Lipstick tree, Transcriptome, Bixin synthesis, Carotenoids

\section{Background}

The nutritional and pharmaceutical potential of plant secondary metabolites is vast and still largely unexplored. Many plant species utilized for production of secondary metabolites that are important components of human diet, animal feed, medicines, biopesticides, and bioherbicides, have been subject of limited research and genetic improvement. This is the case of Bixa orellana L., achiote in Mexico, a species belonging to the Bixaceae

\footnotetext{
* Correspondence: renata@cicy.mx

${ }^{1}$ Centro de Investigación Científica de Yucatán, A. C. Calle 43 No. 130, Col.

Chuburná de Hidalgo, 97200 Mérida, Yucatán, Mexico

Full list of author information is available at the end of the article
}

family within the order Malvales [1, 2]. Bixa orellana is a tropical perennial and ligneous plant of great agroindustrial interest due to its high content of bixin, an apocarotenoid located mainly in the seeds. Bixin or annatto is an orange-red pigment that has been used for many years as a dye in foods, such as dairy and bakery products, vegetable oils, and drinks [3]. The world demand for annatto is increasing together with the interest in natural food dyes.

Carotenoids are yellow to red pigments synthesized by microorganisms and plants. In plants, they accumulate in the plastids (chromoplasts) of flowers and fruits. These compounds have antioxidant functions in all 
organisms, including animals and fungi, and play an important role in protecting cells from damage of radicals such as singlet oxygen [4]. Carotenoids are the major source of vitamin A (retinol) in animals, and abscisic acid (ABA) in plants [5]. All carotenoids are synthesized by consecutive condensations of isopentenyl diphosphate (IPP), which in turn is synthesized through the plastidial methylerythritol phosphate (MEP) pathway [6, 7]. Seven enzymatic steps produce IPP from pyruvate and glyceraldehyde-3-phosphate [6,7]. The first step in carotenoid biosynthesis is the head-to-head condensation of two geranylgeranyl diphosphate (GGDP) molecules to produce phytoene, catalyzed by phytoene synthase (PSY). Subsequently, four enzymes convert phytoene to lycopene via phytofluene, zeta-carotene and neurosporene: two desaturases introduce four double bonds (phytoene desaturase (PDS), and zeta-carotene desaturase (ZDS)), and two isomerases acting, respectively, on the 7/9-7'/9' double bound (carotene cis-trans isomerase, CRTISO) and C15-15' double bonds ( $\zeta$-carotene isomerase, Z-IZO) [8, 9]. The cyclization of lycopene denotes a central branch point in the carotenoid biosynthesis pathway, and the relative activity of epsilon-cyclase $(\varepsilon$-LYC) versus beta-cyclase ( $\beta$-LYC) may determine the flow of carotenoids from lycopene to either $\alpha$-carotene or $\beta$-carotene [8].

Apocarotenoids as bixin are derived from the oxidative cleavage of carotenoids, which might occur randomly through photo-oxidation or lipoxygenase co-oxidation [10]. At the same time, the enzymatic cleavage of carotenoids through specific carotenoid dioxygenases (CCDs) has also been proposed [10, 11]. Bixin is derived from the enzymatic cleavage of lycopene [12, 13]. A biosynthetic pathway for bixin has been proposed $[12,14]$ and supported using a heterologous expression system [12]. This identification, however, has not been supported by a full characterization. Three B. orellana cDNAs encoding the enzymes required for bixin synthesis derived from the linear $\mathrm{C}_{40}$ lycopene have been identified: lycopene cleavage dioxygenase $(B o L C D)$, bixin aldehyde dehydrogenase $(B o B A L D H)$ and norbixin methyltransferase (BonBMT) [12].

In spite of the great economic importance of achiote, its transcriptome and the genes from MEP and carotenoid pathways remained uncharacterized. Before this work, we had only access to partial sequences of some genes $[14,15]$ obtained from expressed sequences tags (ESTs) isolated from a subtracted cDNA library made with RNA from immature seed and leaves [14]. The library identified clusters of transcripts corresponding to five genes of the MEP pathway: (1-Deoxy-D-xylulose-5phosphate synthase $(D X S), 1$-Deoxy-D-xylulose-5-phosphate reductoisomerase $(D X R), 2-C$-Methyl-D-erythritol 4-phosphate cytidyltransferase (MCT), 4-Hydroxy-3methylbut-2-en-1-yl diphosphate synthase $(H D S)$ ), the intermediate gene geranylgeranyl diphosphate synthase (GGDS), three genes of the carotenoid pathway (PSY, $P D S, Z D S$ ) and three genes of the bixin pathway (Carotene deoxygenase, aldehyde dehydrogenase and methyl transferase), which were overexpressed in immature seeds compared to leaves [14]. The limited genetic and molecular data available for B. orellana, is attributable in part to its high amounts of polyphenols, pigments and gummy polysaccharides, which complicate nucleic acid purification. To overcome this difficulty, Rodríguez-Ávila and co-workers developed a protocol to isolate total RNA from multiple tissues of $B$. orellana [16] that proved effective for single gene assay expression analysis. Here we leverage it together with high throughput sequencing, to assemble a transcriptome for this plant. We demonstrate its use to identify the MEP, carotenoid and bixin pathway genes.

\section{Results}

De novo sequence assembly of $B$. orellana transcriptome To investigate the MEP, carotenoid, and bixin pathways genes, we sequenced the transcriptome of $B$. orellana using mRNA from young leaves and two different developmental stages of seeds (immature and mature) (Fig. 1). From the isolated mRNA we constructed indexed cDNA
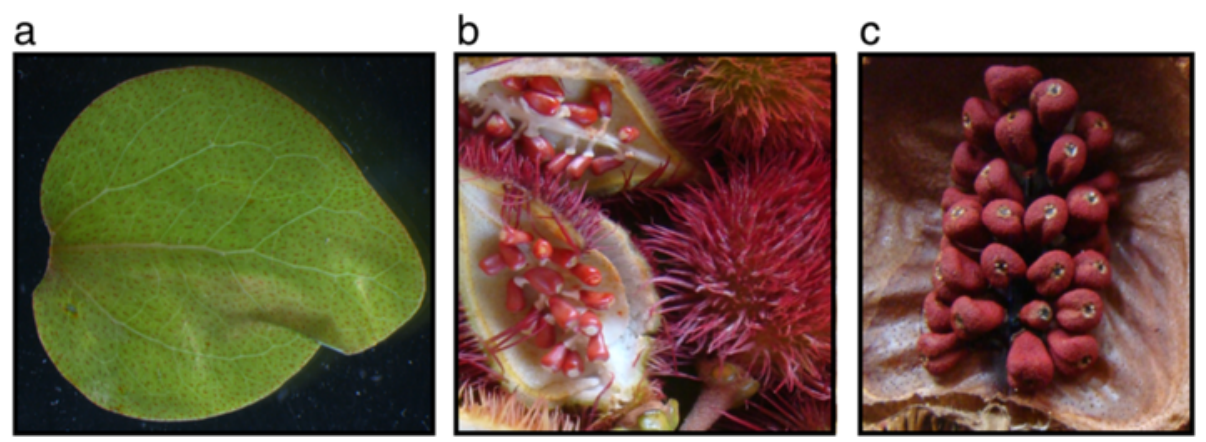

Fig. 1 Bixa orellana tissues used as mRNA sources for sequencing and transcriptome assembly. a Leaf, (b) immature seed, and (c) mature seed 
libraries and sequenced them on the Illumina HiSeq 2500 platform. The reads were assembled de novo using Velvet [17], CLC Genomics Workbench (http://www.clcbio.com) and CAP3 [18] software. In a strategy similar to that of Ashrafi et al., [19], separate Velvet and CLC assemblies were carried out, followed by merging the resulting contigs through CAP3. This strategy optimized the number of different cDNAs assembled, their overall length and the length of the encoded open reading frames (ORF). The final CAP3 set consisted of 52,549 contigs with an N50 of 2,294 bp. The average length of the contigs was 1,924 bp, ranging from 301 to $25,617 \mathrm{bp}$ (Table 1). The contig size distribution showed that 41,209 contigs (78.4 \%) were larger than 1,000 bp, 65 contigs $(0.1 \%)$ had a greater length than $10,000 \mathrm{bp}$ and 11,275 contigs $(21.5 \%)$ were shorter than $1,000 \mathrm{bp}$. Using orf_finder software from WebMGA server [20], we performed an ORF search in order to determine the approximate number and size of the proteins coded in the transcriptome. A total of 25,555 ORFs $\geq 300$ $\mathrm{b}$ were detected, the average length was $1,578.5 \mathrm{~b}$ and the longest had 11,322 b (Table 1).

\section{Evolutionary relationship of Bixa orellana}

In order to elucidate the evolutionary relationship of B. orellana, a phylogenetic analysis of 13 proteins encoded by presumed single-copy genes in most plants, identified by Duarte and co-workers [21], was carried out. These single-copy genes yielded wellresolved tree topologies [21, 22]. The phylogenetic analysis grouped achiote in the Malvidae clade, in close relationship with cotton (Gossypium raimondii) and cacao (Theobroma. cacao) (Fig. 2a).

\section{Blast search in public databases}

We compared achiote transcriptome (52,549 contigs) to three protein databases, NCBI Plant Protein Reference sequence (RefSeq), Phytozome, and PLAZA 3.0, using the BLASTX algorithm with a cutoff e-value of $1 \mathrm{e}-6$. The search against RefSeq exhibited a total of 47,894

Table 1 Assembly statistics

\begin{tabular}{ll}
\hline Total number of contigs & 52,549 \\
Transcriptome size(nt) & $101,106,695$ \\
Longest contig & 25617 \\
Shortest contig & 301 \\
Average contigs length(nt) & 1,924 \\
N50(nt) & 2,294 \\
Total number of ORF & 25,555 \\
Average ORF length(nt) & 1578.5 \\
Longest ORF(nt) & 11,322 \\
Shortest ORF(nt) & 300 \\
\hline
\end{tabular}

The assemblathon_stats perl scripts version 2 and ORF_finder were used to compute assembly statistics contigs (91\%) with significant hits, while comparisons against the Phytozome and PLAZA 3.0 databases showed that 46,232 contigs ( $88 \%$ ) and 48,047 contigs (91\%) had significant hits, respectively. BLAST hits from the RefSeq comparison were distributed between 28 plant species. Eight plant species had $\geq 1 \%$ transcriptome contigs hits (Fig. 3a). Hits obtained by the Phytozome comparison were distributed between 35 plant species; ten of them had $\geq 1 \%$ transcriptome contigs blast hit (Fig. 3b). Twentyeight plant species were represented in the 48,045 BLAST hits obtained by PLAZA 3.0 comparison, and 9 out of the $28 \mathrm{had} \geq 1 \%$ transcriptome contigs blast hit (Fig. 3c). In all comparisons, cacao (T. cacao) provided the best BLAST hits: 33,442 contigs (64. \%) when the transcriptome was compared with the RefSeq database, 27,454 contigs (52\%) compared with the Phytozome database and 27,362 contigs (52\%) with the PLAZA 3.0 database (Fig. 3). The second best represented plant species in the BLAST results was orange (Citrus sinensis) with 2446 contigs from the RefSeq comparison and cotton (G. raimondii) with 6016 and 6410 contigs displayed by Phytozome and PLAZA 3.0 comparisons, respectively (Fig. 3). BLASTX results for transcriptome comparisons are available in Additional file 1: Table S1.

To compare the achiote transcriptome with a previous achiote EST library created by Jako and co-workers [14], we performed a bidirectional BLASTN. Jako and coworkers library has 954 sequences registered, the longest sequence is $691 \mathrm{bp}$ and the shortest is $50 \mathrm{bp}$ with a mean sequence size of $355 \mathrm{bp}$ [14]. Using the EST library as a query, we found that 714 EST sequences $(74.8 \%)$ had BLAST hits, with an average identity of $99 \%$ and identity range between 90.91 and $100 \%$ (Additional file 1: Table S2). Whereas, 583 contigs (1.1\% of transcriptome) had hits to the EST library, with a high average identity of $98.6 \%$. The identity range was between $82.77 \%$ and $100 \%$ (Additional file 1: Table S2).

\section{Functional annotation of gene ontology}

We used the BLASTX results of the achiote transcriptome against the RefSeq database to extract Gene Ontology (GO) terms with Blast2GO software. 38,076 contigs $(80 \%)$ with significant hits out of 47,894 were annotated and classified in 7461 GO terms. These GO terms were split in the three main GO categories, "Biological process" (4314 Go terms), "Molecular function" (2485 terms) and "Cellular component" (665 GO terms). In "biological process", the top three GO descriptions from level 2 were "cellular process" with 22,066 contigs, "metabolic process" with 21,664 contigs and "single-organism process" with 20,762. In "molecular function", the largest description was "catalytic activity" with 17,260 contigs followed by "binding" and "transporter activity". In reference to the "cellular component" term, 


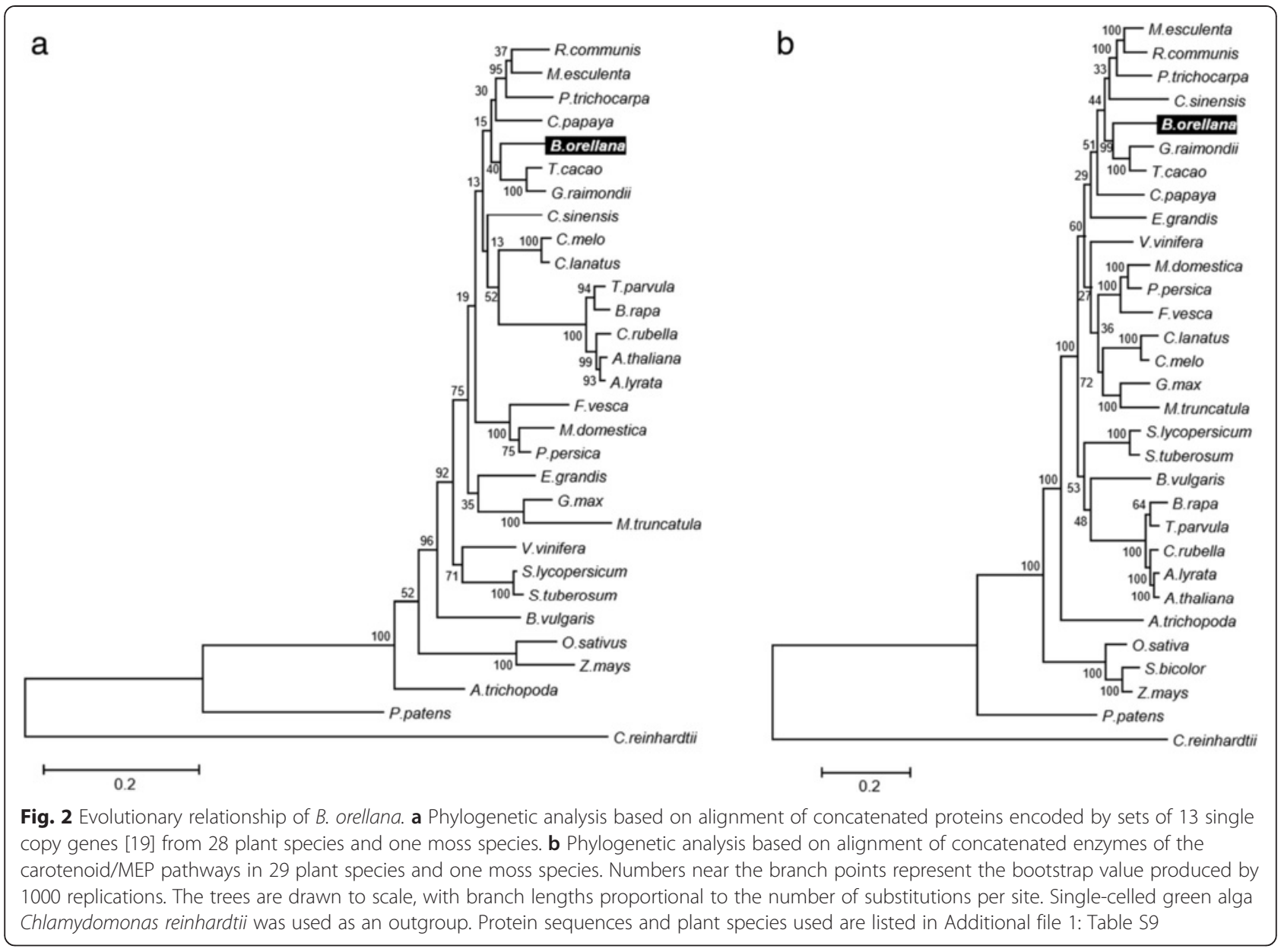

the most represented descriptions were "cell", "organelle" and "membrane" with 29,327, 23,421 and 11,200 contigs, respectively (Fig. 4a).

With regard to carotenoids biosynthesis, 601 contigs from 38,076 with GO annotation were classified in "terpenoid metabolic process" (GO:0006721, Fig. 4b). 369 contigs (61.4\%) from this description belong to GO term "carotenoid" (GO:0016117). The rest of 232 contigs included in GO:0006721 were split in three descriptions, "diterpenoid", "triterpenoid", and "sesquiterpenoid". GO annotation is available in Additional file 1: Table S3.

\section{KEGG pathway annotation}

In order to assign biochemical pathways to B. orellana transcriptome, a functional pathway annotation was performed against the Kyoto Encyclopedia of Genes and Genomes (KEGG). The KEGG annotation was carried out with the KAAS server (KEGG Automatic Annotation Server) by BLAST comparisons against the KEGG GENES database. When the file with 52,549 contigs of transcriptome was uploaded to the server, 8698 were assigned to 3092 enzymes. The five main KEGG biochemical pathways were represented: metabolism (2349 contigs), genetic information processing (2082), organism system (851), cellular processes (764) and environmental information processing (783). In metabolism pathways, 2349 contigs were distributed in 5058 hits (Fig. 5a). The top three groups of metabolism pathways were "carbohydrate metabolism" with 1021 hits against 190 enzymes, followed by "amino acid metabolism" with 700 hits in 183 enzymes. The third group called "overview", which included Carbon metabolism, 2Oxocarboxylic acid metabolism, Fatty acid metabolism, Biosynthesis of amino acids and Degradation of aromatic compounds), had 506 hits and 175 enzymes.

In the terpenoids and polyketides pathways, which include the carotenoid pathways, 175 contigs could be associated with 75 enzymes (Fig. 5b). The largest pathway with 48 contigs was "Terpenoid backbone biosynthesis", which includes enzymes from the MEP and mevalonate pathways. The carotenoid pathway was the second most represented group with 38 contigs and 17 enzymes. The twelve enzymes belonging to the carotenoid pathway were: PSY, PDS, 15-Z-ISO, ZDS, CRTISO, $\beta$-LYC, $\varepsilon-$ LYC, $\beta$-carotene hydroxylase $(\beta \mathrm{CH})$, cytochrome P450type monooxygenase 97A (CYP97A3), cytochrome 

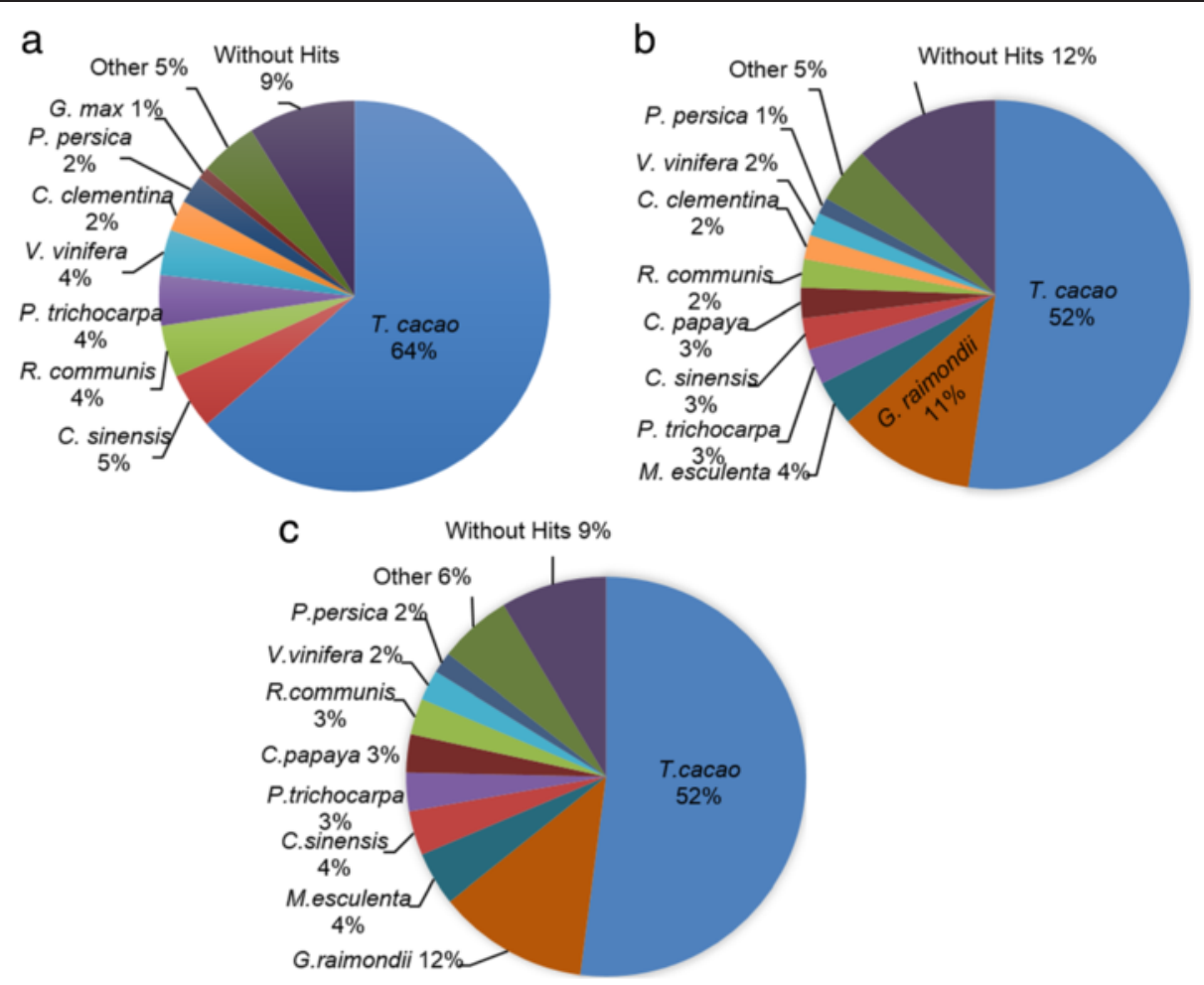

Fig. 3 BLASTX top-hits species distribution. The B. orellana transcriptome was compared to: (a) the NCBI RefSeg plant protein database, (b) the Phytozome protein database version 10, and (c) the PLAZA protein database version 3.0. The percent of contigs producing hit for each species is marked after the species scientific name

P450-type monooxygenase 97C1 (CYP97C1), zeaxanthin epoxidase (ZEP) and violaxanthin de-epoxidase (VDE). The five remaining enzymes are associated to derivate compounds of carotenes: capsanthin/capsorubin synthase (CCS1), 9-cis-epoxycarotenoid dioxygenase (NCED), xanthoxin dehydrogenase (ABA2), abscisic-aldehyde oxidase (AAO3) and abscisic acid 8' -hydroxylase. KEGG annotation is available in Additional file 1: Table S4.

\section{Identification of MEP and carotenoid pathways CDNAs from $B$. orellana transcriptome}

To identify and isolate the cDNAs encoding proteins of the MEP and carotenoid pathway, a Local TBLASTN search against the achiote transcriptome was performed using homologous proteins from Arabidopsis thaliana, G. raimondii and $T$. cacao followed by a phylogenetic analysis of each putative protein. The search allowed us to identify the cDNAs encoding the seven canonical enzymes in the MEP pathway, the cDNAs encoding the 14 core enzymes of the carotenoid pathways and the cDNAs encoding intermediate pathway proteins isopentenyl diphosphate isomerase (BoIDI) and BoGGDS (Table 2).

cDNAs encoding putative BoDXS in the MEP pathway were consistent with four genes: BoDXS1, BoDXS3 and two paralogous copies of BoDXS2 (BoDXS2a and $B o D X S 2 b)$. We identified cDNA consistent with single copy genes for the remaining MEP pathway enzymes: BoDXR, BoMCT, 4-Diphosphocytidyl-2-C-methyl-Derythritol kinase $(B o C M K)$, 2-C-Methyl-D-erythritol 2,4-cyclodiphosphate synthase (BoMDS), BoHDS, and 4-Hydroxy-3-methylbut-2-enyl diphosphate reductase $(B o H D R)$. Also single copies were identified for the intermediate genes BoIDI and BoGGPS. Comparison to MEP pathways cDNAs isolated in the previous EST library [14] showed that BoDXS2a, BoDXR1, BoCMK, BoHDS, BoHDR and BoGGDS were common (Table 2).

In the carotenoid pathway, cDNAs characterization identified two gene copies for phytoene synthase (BoPSY1 and BoPSY2), phytoene desaturase (BoPDS1 and BoPDS2), $\beta$ lycopene cyclase $(B o \beta-L Y C 1$ and $B o \beta-L Y C 2)$, zeaxanthin epoxidase (BoZEP1 and BoZEP2) and violaxanthin deepoxidase (BoVDE1 and BoVDE2). The remaining carotenoid pathway genes were found in single copy, except CRTISO for which three copies were identified: $B o C R$ TISO2 and paralogous BoCRTISO1a and BoCRTISO1b (Table 2). The comparison between carotene pathway cDNAs isolated in the Jako and co-workers library [14] showed that only the cDNAs encoding BoPSY1, BoPSY2, BoPDS1 and BoZDS were in common (Table 2). 


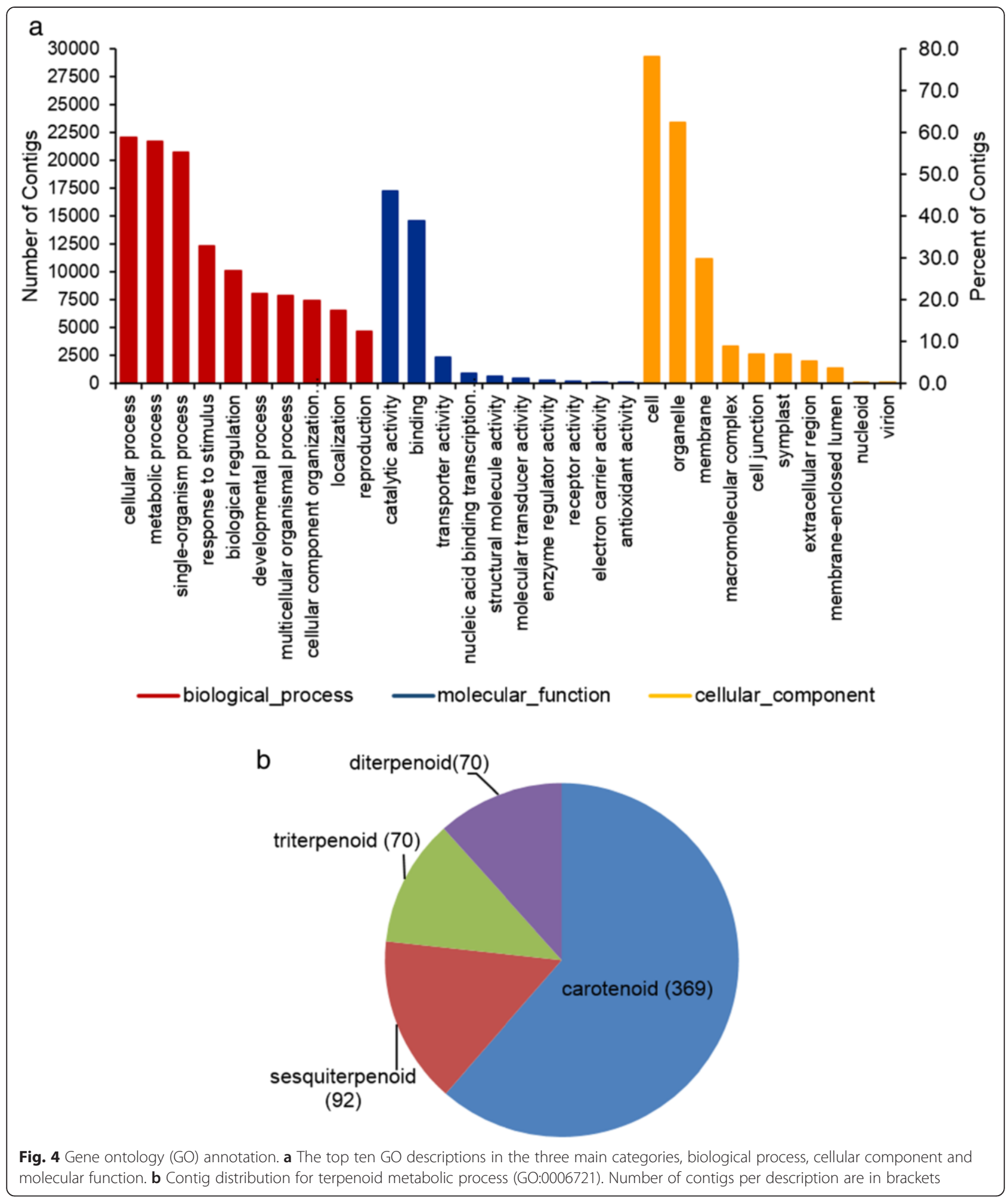

In order to elucidate the evolutionary relationship of MEP and carotenoid pathways enzymes from $B$. orellana and other plant species, we carried out a phylogenetic analysis using MEGA6 software. The analysis was based on alignment of concatenated protein sequences from
MEP and carotenoid pathways of B. orellana and 27 plants species. B. orellana was grouped with species from the Malvidae clade and was closely related to cotton and cacao, the two Malvales species available in sequence databases (Fig. 2b). 


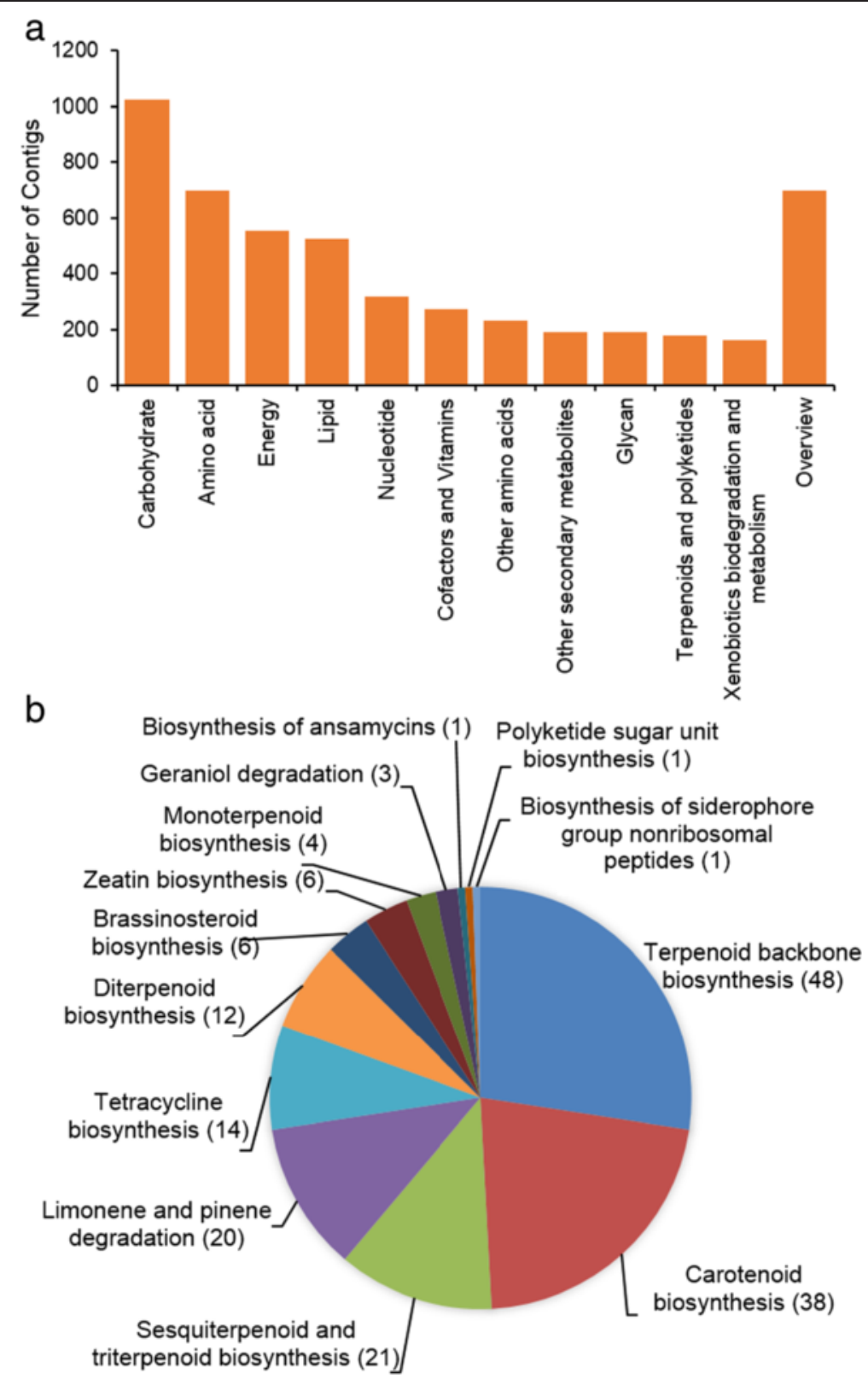

Fig. 5 Kyoto Encyclopedia of Genes and Genomes (KEGG) annotation. a Classification based on metabolism categories. b Classification based on metabolism of terpenoids and polyketides. Number of contigs per pathway is in brackets

\section{Identification of new genes in bixin pathways}

To identify and isolate the cDNAs encoding enzymes of the bixin pathway, a TBLASTN search against the achiote transcriptome was performed using the achiote protein sequences previously reported by Bouvier and co-workers (BoLCD, [GenBank: AJ489277]; BoBADH, [GenBank: AJ548846]; BonBMT, [GenBank: AJ548847]) [12]. Surprisingly, these three proteins were not present among those encoded by our assembled transcriptome. The Bouvier BoLCD protein had only one hit with $53 \%$ of identity. BoBADH displayed hits with seven contigs with low identity percentages (49-52 \%). When BonBMT was compared, several hits with identity range between 35 and $49 \%$ were found. On the other hand, our previously described CCD1 [13] matched several contigs with high identity (75-98\%). We were also able to identify high quality matches in $B$. orellana for cDNAs encoding carotenoid cleavage dioxygenase 4 (CCD4), aldehyde dehydrogenases (ALDHs) and carboxyl methyltransferases using homologous proteins of $A$. thaliana and $T$. cacao. 
Table 2 Identified CDNA from MEP, carotenoid and bixin pathways

\begin{tabular}{|c|c|c|}
\hline Description & $\begin{array}{l}\text { Jako } \\
\text { Hits }\end{array}$ & $\begin{array}{l}\text { GenBank } \\
\text { Accession }\end{array}$ \\
\hline $\begin{array}{l}\text { BoDXS1 (1-Deoxy-D-xylulose-5-phosphate } \\
\text { synthase) }\end{array}$ & 0 & KT358983 \\
\hline BoDXS2a & 2 & KT358984 \\
\hline BoDXS2b & 0 & KT358985 \\
\hline BoDXS3 & 0 & KT358986 \\
\hline $\begin{array}{l}\text { BoDXR (1-Deoxy-D-xylulose-5-phosphate } \\
\text { reductoisomerase) }\end{array}$ & 2 & KT358987 \\
\hline $\begin{array}{l}\text { BoMCT (2-C-Methyl-D-erythritol 4-phosphate } \\
\text { cytidyltransferase) }\end{array}$ & 0 & KT358988 \\
\hline $\begin{array}{l}\text { BoCMK ( 4-Diphosphocytidyl-2-C-methyl- } \\
\text { D-erythritol kinase) }\end{array}$ & 1 & KT358989 \\
\hline $\begin{array}{l}\text { BoMDS (2-C-Methyl-D-erythritol 2,4- } \\
\text { cyclodiphosphate synthase) }\end{array}$ & 0 & KT358990 \\
\hline $\begin{array}{l}\text { BoHDS 4-Hydroxy-3-methylbut-2-en-1-yl } \\
\text { diphosphate synthase) }\end{array}$ & 3 & KT358991 \\
\hline $\begin{array}{l}\text { BoHDR (4-Hydroxy-3-methylbut-2-enyl } \\
\text { diphosphate reductase) }\end{array}$ & 1 & KT358992 \\
\hline BoIDI (Isopentenyl diphosphate isomerase) & 0 & KT358993 \\
\hline BoGGDS (Geranylgeranyl diphosphate synthase) & 4 & KT358994 \\
\hline BoPSY1 (Phytoene synthase) & 1 & KT358995 \\
\hline BoPSY2 & 1 & KT358996 \\
\hline BoPDS1 (Phytoene desaturase) & 9 & KT358997 \\
\hline BoPDS2 $^{\mathrm{a}}$ & 0 & KT358998 \\
\hline BoZ-ISO (15-cis-Z-carotene isomerase) & 0 & KT358999 \\
\hline BoZDS (Z-carotene desaturase) & 8 & KT359000 \\
\hline BoCRTISO1a (Carotene cis-trans isomerase) & 0 & KT359001 \\
\hline BoCRTISO1b & 0 & KT359002 \\
\hline BOCRTIOS2 & 0 & KT359003 \\
\hline Boß-LYC1 (Lycopene $\beta$-cyclase) & 0 & KT359004 \\
\hline Boß-LYC2 & 0 & KT359005 \\
\hline Boz-LYC (Lycopene $\varepsilon$-cyclase) & 0 & KT359006 \\
\hline BoßCH1 ( $\beta$-carotene hydroxylase) & 0 & KT359007 \\
\hline $\begin{array}{l}\text { BoCYP97A3 (Cytochrome P450-type } \\
\text { monooxygenase 97A3) }\end{array}$ & 0 & KT359008 \\
\hline $\begin{array}{l}\text { BoCYP97C1 (Cytochrome P450-type } \\
\text { monooxygenase 97C1) }\end{array}$ & 0 & KT359009 \\
\hline $\begin{array}{l}\text { BoCYP97B3 }{ }^{\text {a }} \text { (Cytochrome P450-type } \\
\text { monooxygenase 97B3) }\end{array}$ & 0 & KT359010 \\
\hline BoZEP1 (Zeaxanthin epoxidase) & 0 & KT359011 \\
\hline BoZEP2 & 0 & KT359013 \\
\hline BoVDE1 (Violaxanthin de-epoxidase) & 0 & KT359014 \\
\hline BoVDE2 $^{a}$ & 0 & KT359015 \\
\hline BoNSY (Neoxanthin synthase) & 0 & KT359016 \\
\hline BoCCD1-1 (Carotene cleavage dioxygenase 1-Copy1) & 0 & KT359018 \\
\hline BoCCD1-2 & 0 & KT359019 \\
\hline BoCCD1-3 & 0 & KT359020 \\
\hline BoCCD1-4 ${ }^{a}$ & 0 & KT359021 \\
\hline
\end{tabular}

Table 2 Identified cDNA from MEP, carotenoid and bixin pathways (Continued)

\begin{tabular}{|c|c|c|}
\hline $\begin{array}{l}\text { BoCCD4-1 (Carotene cleavage dioxygenase } \\
\text { 4-Copy1) }\end{array}$ & 0 & KT359022 \\
\hline BoCCD4-2 & 9 & KT359023 \\
\hline BoCCD4-3 & 16 & KT359024 \\
\hline BoCCD4-4 & 0 & KT359025 \\
\hline BoCCD4-5 $5^{a}$ & 0 & KT359026 \\
\hline BoALDH2B4 (aldehyde dehydrogenase 2B4) & 0 & KT359027 \\
\hline BoALDH2B7-1 & 0 & KT359028 \\
\hline BoALDH2B7-2 & 0 & KT359029 \\
\hline BoALDH2C4 ${ }^{a}$ & 0 & KT359030 \\
\hline BoALDH3F1 & 0 & KT359031 \\
\hline BoALDH3F2 & 0 & KT359032 \\
\hline BoALDH3H1-1 & 10 & KT359033 \\
\hline BoALDH3H1-2 & 0 & KT359035 \\
\hline BoALDH3I1 & 2 & KT359036 \\
\hline BoALDH5F1 & 0 & KT359038 \\
\hline BoALDH6B2-1 & 0 & KT359039 \\
\hline BoALDH6B2-2 & 0 & KT359040 \\
\hline BoALDH6B3 & 0 & KT359041 \\
\hline BoALDH7B4 & 1 & KT359042 \\
\hline BoALDH10A8 & 0 & KT359043 \\
\hline BoALDH11A3 & 0 & KT359044 \\
\hline BoALDH12A1 & 0 & KT359045 \\
\hline BoALDH18B1-1 & 0 & KT359046 \\
\hline BoALDH18B1-2 & 0 & KT359047 \\
\hline BoALDH22A1 & 0 & KT359048 \\
\hline $\begin{array}{l}\text { BoSABATH1 (SABATH family } \\
\text { Methyltransferase1) }\end{array}$ & 0 & KT359049 \\
\hline BOSABATH2 & 0 & KT359050 \\
\hline BOSABATH3 & 3 & KT359051 \\
\hline BOSABATH4 & 6 & KT359052 \\
\hline BOSABATH5 & 0 & KT359053 \\
\hline BOSABATH6 & 0 & KT359054 \\
\hline BOSABATH7 & 0 & KT359055 \\
\hline BOSABATH8 & 0 & KT359056 \\
\hline BOSABATH9 & 0 & KT359057 \\
\hline BoSABATH $10^{a}$ & 0 & KT359058 \\
\hline BOSABATH11 & 0 & KT359059 \\
\hline BoSABATH12 & 0 & KT359060 \\
\hline
\end{tabular}

${ }^{\mathrm{a} P a r t i a l}$ sequence

Carotenoid cleavage dioxygenase proteins in bixin synthesis The contigs similar to the CCD1 isolated by RodríguezÁvila and co-workers [13], allowed the identification of three paralogous copies of the CCD1 gene (BoCCD1-2, $B o C C D 1-3$ and BoCCD1-4). A pair-wise comparison 
between CCD1 protein sequences showed that the BoCCD1 described by Rodríguez-Ávila and co-workers [13] shared $96.9 \%$ identity with BoCCD1-2, $75 \%$ with BoCCD1-3 and $75 \%$ with BoCCD1-4 (Additional file 1: Table S5). Additionally, another CCD1 sequence was identified by PCR when $B o C C D 1-2$ sequences were amplified and characterized for corroboration. This new cDNAs probably corresponds to an allele of BoCCD1-2 because it shared $97 \%$ of nucleotide identity. The gene was called BoCCD1-1. BoCCD1-1 protein shared $98 \%$ identity with the CCD1 isolated by Rodríguez-Ávila and co-workers [13] and $95 \%$ with BoCCD1-2 (Additional file 1: Table S5). No BoCCD1 genes were reported by Jako and co-workers (Table 2) [14]. Comparison of CCD4 homologous proteins against those encoded by the assembled achiote transcriptome allowed us to identify five BoCCD4 genes (BoCCD4-1, BoCCD4-2, BoCCD4-3, BoCCD4-4, and BoCCD4-5). The pair-wise comparison between these proteins exhibited an identity range between 47 to $67 \%$ (Additional file 1 : Table S5). The previous CCD4 isolated by Bouvier and co-workers [12] displayed low identity (30-35\%) in comparison with the proteins coded by our transcriptome (Additional file 1: Table S5). Of the five $B o C C D 4$ cDNAs characterized in this work, BoCCD42 and BoCCD4-3 matched EST sequences from Jako and co-workers library (Table 2) [14].

Phylogenetic analysis of BoCCDs proteins yielded two major clades; BoCCD1 and BoCCD4 clustered with the CCD1 and CCD4 families, respectively. BoCCD1-1 and -2 were closely related to the BoCCD1 described by Rodríguez-Ávila and co-workers [13]. BoCCD1-1 and -2 clustered with monocotyledonous CCD1 proteins, albeit with poor bootstrap support. BoCCD1 copy 3 and copy 4 were not closely related to the BoCCD1 protein described by Rodríguez-Âvila and co-workers [13], but grouped together outside the major CCD1 clade (Additional file 2: Figure S1). With regard to the BoCCD4 proteins, BoCCD4-1, -2, -3 and -4 are grouped together (Additional file 2: Figure S1). The small BoCCD4 family clustered in a subclade of CCD4 proteins from woody plants such as T. cacao, Vitis vinifera, and Populus trichocarpa. The incomplete sequence of BoCCD4-5, suggests a more distant relationship to the BoCCD4 small family defined by the previous proteins. BoCCD4- 5 is related to the CCD4 from Ricinus communis, P. trichocarpa, T. cacao and $G$. raimondii grouped in the other CCD4 subclade (Additional file 2: Figure S1). The BoLCD sequence described by Bouvier and co-workers [12] was not closely related to BoCCD4 proteins found in this work, but grouped instead in the monocotyledonous CCD4 clade, close to three CCD4 from monocotyledonous Crocus sativus (Additional file 2: Figure S1). This latter clade's strong support (99\% bootstrap value) suggests that their previous attribution to B. orellana by Bouvier and coworkers [12] is spurious.

\section{Aldehyde dehydrogenase proteins}

To identify cDNAs encoding BoALDHs, we performed TBLASTN search using T. cacao and A. thaliana homologous ALDH proteins from the 13 distinct ALDH families of plants. This approach succeeded in identifying 20 different ALDHs cDNAs. According to the phylogenetic analysis of BoALDH and its homologous proteins, the BoALDHs isolated in this work belong to 10 ALDH families (Table 2 and Additional file 2: Figure S2). Four BoALDH proteins were clustered in the ALDH2 family, five with ALDH3, three with ALDH6 and two with ALDH18. The remaining BoALDH proteins grouped with the ALDH5, ALDH7, ALDH10, ALDH11, ALDH12 and ALDH22 families (Table 2 and Additional file 2: Figure S2). BoBADH described by Bouvier and co-workers [12] was more distant to BoALDHs, and closer to the protein from monocotyledonous Crocus sativus in subfamily ALDH2C4 (Additional file 2: Figure S2), another possible spurious instance. BoALDH3H1-1, BoALDH3I and BoALDH7B4 genes yielded BLAST hits with 10, 2 and 1 sequences respectively in the Jako and coworkers library [14] (Table 2).

\section{Methyltransferases proteins}

In order to identify carboxyl methyltransferase proteins encoded by $B$. orellana transcriptome, we used T. cacao and $A$. thaliana homologous proteins belonging to the SABATH methyltranferase family (plant proteins with the ability to methylate carboxyl groups [23]) to perform a TBLASTN search. We found 12 different proteins (Table 2 and Additional file 2: Figure S3). Phylogenetic analysis of SABATH proteins divided them in three major clades called I, II and III (Additional file 2: Figure S3), which, however, differed from a previous phylogenetic classification [23]. BoSABATH1, BoSABATH2 and a small group of four BoSABATH proteins (BoSABATH 3, 4, 5 and 6) were grouped in Clade I. Also, the previously described BonBMT was grouped in this clade, but was not closely related to our BoSABATH protein. Instead, it displayed high similarity to a $C$. sativus carboxyl methyltransferase. This clade's strong support (96 \% bootstrap value) suggests another spurious instance of BonBMT described by Bouvier and co-workers [12] (Additional file 2: Figure S3). BoSABATH2 was the only one grouped in the small clade II, for which most members are jasmonic acid carboxyl methyltransferases. In clade III, BoSABATH10 was grouped in a subclade formed by ten $A$. thaliana SABATH proteins. Additionally, BoSABATH7, 8, 9, 11 and 12 were clustered in clade III and a small BoSABATH group was formed by BoSABATH8, 11 and 12 (Additional file 2: Figure S3). BoSABATH3 and BoSABATH4 proteins matched, 
respectively, 3 and 6 sequences in Jako and co-workers library [14] (Table 2).

\section{Gene expression of selected carotenoid and bixin pathway key genes}

We selected key cDNAs of the carotenoid and bixin biosynthesis pathways for qRT-PCR quantification of their transcript levels in new RNA samples from leaves, immature seeds and mature seeds (Fig. 6). In the MEP pathway, we found that BoDXS2a was overexpressed in immature seed in comparison to mature seed and leaf (Fig. 6). In the carotenoid pathway, we select BoPSY1, BoPSY2, BoPDS1, BoZDS Boß-LYC1, Boß-LYC2 and Boe$L Y C$ for qRT-PCR quantification. BoPDS1 and BoZDS were up-regulated in immature seed whereas BoPSY1, $B o P S Y 2, \quad B o \beta-L Y C 1, \quad B o \beta-L Y C 2$ and Boc-LYC were expressed preferentially in leaf (Fig. 6). In the bixin pathway, we selected 14 cDNAs, four BoCCD1 (BoCCD1-1 to -4 ), four BoCCD4 (BoCCD4-1 to -4), three BoALDH3 (BoALDH3F1, BoALDH3H1 and BoALDH3I1) and three BoSABATH (BoSABATH1, BoSABATH3 and BoSABATH4). BoCCD1-1, BoCCD4-4 and BoALDH3F1 displayed no changes in transcript levels between leaf and immature seed, whereas the remaining genes showed differential expression levels. Amongst these differential expressed genes, ten were up-regulated in immature seeds and one was up-regulated in leaves (BoCCD1-2) (Fig. 6). In all cases the lowest expression levels were displayed in mature seed (Fig. 6). The oligonucleotides sequences used as primers are listed in Additional file 1: Table S6.

\section{Discussion}

Achiote plants are the source of bixin apocarotenoid. Therefore, identification in this species of the genes encoding the putative enzymes of the pathways contributing to bixin synthesis, such as MEP, carotenoid and bixin pathways, is of basic and applied importance. Description of these genes before this study was limited and incomplete $[12-15,24,25]$, probably due to coverage limitation of the available EST libraries from immature seeds $[14,25]$. A complicating factor is that $B$. orellana is recalcitrant to molecular biology studies, probably because its tissues contain high amounts of secondary metabolites that hinder purification of nucleic acids [16]. With development of high throughput sequencing technology, which are effective with lesser amounts and shorter fragments of RNA, whole transcriptome sequencing became feasible in B. orellana. This technology has successfully been applied to identify

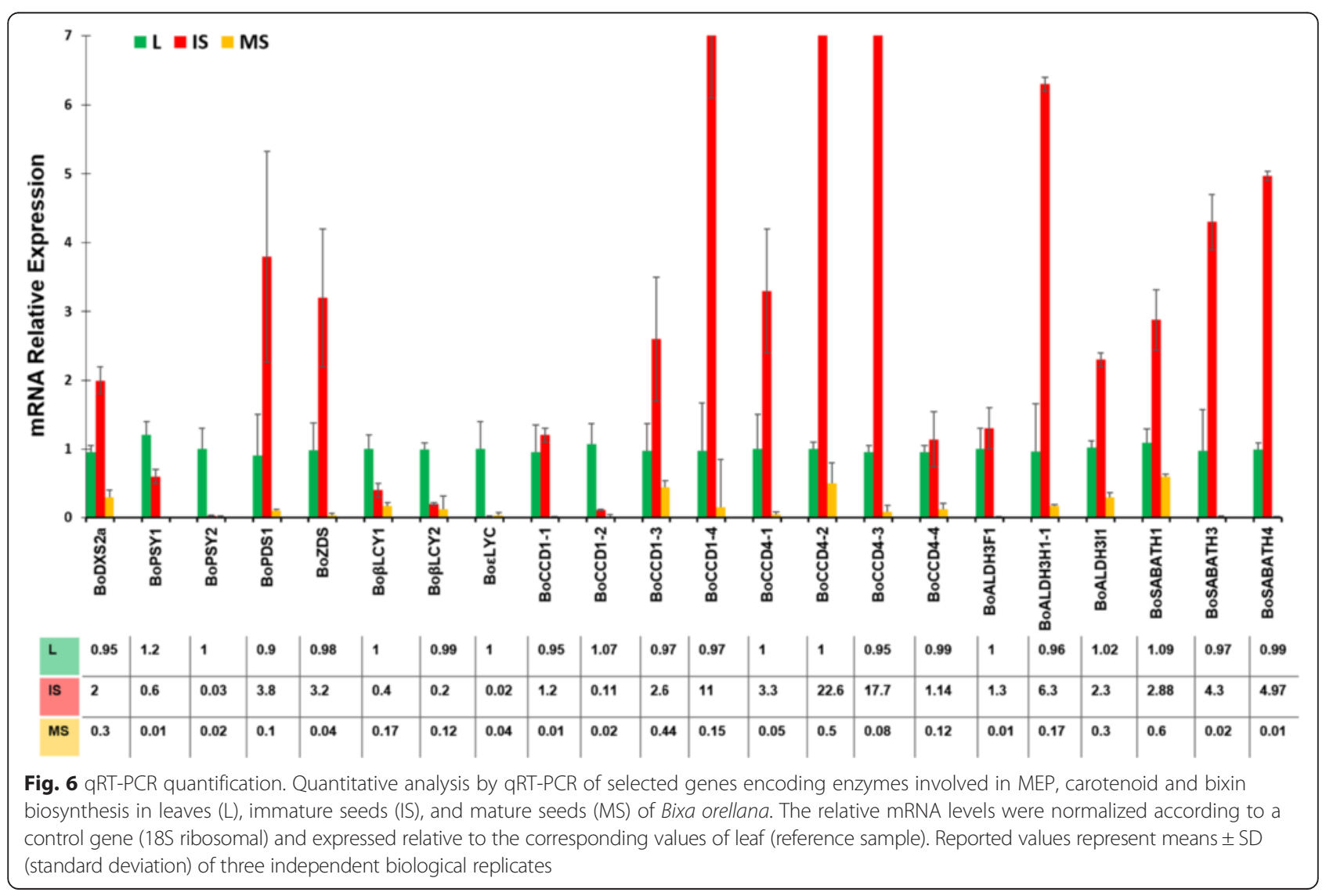


the MEP and carotenoid pathways genes in Momordica cochinchinensis [26], Citrus sinensis [27] and Citrullus lanatus [28]. Application of this technology to sequencing the first $B$. orellana transcriptome allowed us to elucidate the complete bixin biosynthesis pathway including MEP and carotenoid pathways.

\section{Transcriptome assembling of Bixa orellana}

A total of 52,549 contigs were obtained from the transcriptome assembly, which was carried out with the combined use of three assembly programs, Velvet, CLC and CAP3, each providing complementary strengths [19]. A total of 25,555 proteins larger than 100 aa were predicted in the achiote transcriptome, a number similar to that of other sequenced species such as T. cacao, C. papaya, $C$. sinensis, C. clementina and V. vinifera [29-32]. BLAST comparison of this transcriptome with the existing B. orellana library database [14] and 21 homologous proteins previously isolated [12, 13,33-37], confirmed that our $B$. orellana assembly is reliable because of high coverage and identity (Additional file 1: Table S2 and Table S7). Moreover, the cDNA sequence covering predicted full length ORFs of carotenoid (BoPSY1, BoPSY2, BoPDS1, BoZ-ISO, BoZDS, BoCRTISO1, BoCRTISO2 and BoßLYC1) and bixin (Five $B o C C D 1 s$ and four BoCCD4s) pathways genes obtained through the in silico assembly were confirmed by independent cDNA sequencing.

\section{Evolutionary relationship of Bixa orellana}

According to the Angiosperm Phylogeny Group (APG) system, B. orellana belongs to the Malvales order, Malvidae clade. Malvales include several commercial crops such as kenaf (Hibiscus cannabinus), roselle (Hibiscus sabdariffa), cacao (Theobroma cacao), cotton (species of Gossypium) and cola nut (Cola acuminata) [1, 2]. Phylogenetic reconstructions based on two sets of $B$. orellana proteins (13 general proteins encoded by single copy genes [21] and additional selected proteins of the carotenoid/MEP pathways) is in agreement with APG classification. As shown in Fig. 2, B. orellana is grouped with two members of Malvales available in sequence databases ( $T$. cacao and G. raimondii). Interestingly, this small group is more closely related to members of the order Malpighiales ( $R$. communis M. esculenta and P.trichocarpa) than to other orders of Malvidae such as Brassicales or Huertelaes. This discrepancy has been documented, suggesting that the order Malpighiales belongs to the Malvidae rather than Fabidae [38, 39]. The evolutionary relationship of $B$. orellana with Malvales and Malpighiales is also reflected in the comparison of the whole achiote transcriptome against plant protein databases (Fig. 3). As shown in Fig. 3 cacao is most represented among the matches in the Phytozome and Plaza 3.0 comparisons, followed by cotton (G. raimondii), cassava ( $M$. esculenta), citrus (C. sinensis), poplar (P. trichocarpa), papaya (C. papaya) and castor bean ( $R$. communis). Comparison to RefSeq was biased because most proteins of G. raimondii, M.esculenta and C. papaya were not available there through May, 2014.

\section{Methylerythritol phosphate (MEP) pathway genes}

The MEP pathway is the predominant supplier of carotenoid biosynthesis precursors isopentenyl and dimethylallyl diphosphate (IPP and DMAPP) [40]. In this pathway, pyruvate and glyceraldehyde 3-phosphate are condensed and converted to IPP and DMAPP by seven enzymes (DXS, DXR, MCT, CMK, MDS, HDS and HDR). In this work, we identified the genes encoding these enzymes (Table 2 and Fig. 7). Similar to species with multi-copies of DXS gene [28, 41, 42], achiote also has a small family of four BoDXS genes. Phylogenetic analysis of DXS proteins grouped one protein in the DXS type I clade (BoDXS1), two proteins in the DXS type II clade (BoDXS2a and BoDXS2b) and the last (BoDXS3) in the DXS type III clade (Additional file 2: Figure S4). Enzymes from the DXS2 clade, but not DXS1 or DXS3, are involved in carotenoid and apocarotenoid accumulation in non-photosynthetic tissues like seeds $[41,43,44]$. In this work, we found that the BoDXS2a gene was overexpressed in immature seeds (Fig. 6), which suggests that BoDXS2a could be involved in the synthesis of seed carotenoids and apocarotenoids. Overexpression in immature seed of BoDXS2a (this work), and BoDXR, BoHDS and BoHDR (Table 2) [14], might lead to high concentration of carotenoids and apocarotenoids in immature seed.

\section{Carotenoid pathway genes of Bixa orellana}

The carotenoid biosynthetic pathway includes 14 enzymes that convert two GGDP molecules into a variety of carotenoids. Here, we infer from cDNA characterization the existence of 21 genes encoding these enzymes (Table 2 and Fig. 7). With the exception of BoPSY, the qRT-PCR quantification profiles suggest enhanced lycopene production in immature seeds, analogous to what was observed during red ripening in tomato fruits. The accumulation of lycopene in tomato is apparently due to downregulation of $\beta$-LYC and $\varepsilon-L Y C$, and upregulation of PSY, PDS and $Z D S$ [45-49]. Positive feedback regulation may occur during tomato ripening: expression of $P D S$ and $Z D S$ increases in response to low quantities of endproducts of the carotenoid pathway, such as $\beta$ carotene, xanthophylls or ABA $[49,50]$. A similar scenario could take place in immature seed of B. orellana: genes that encode cyclase enzymes were downregulated in immature seed (Fig. 6), potentially blocking the carotenoid pathway below lycopene and 


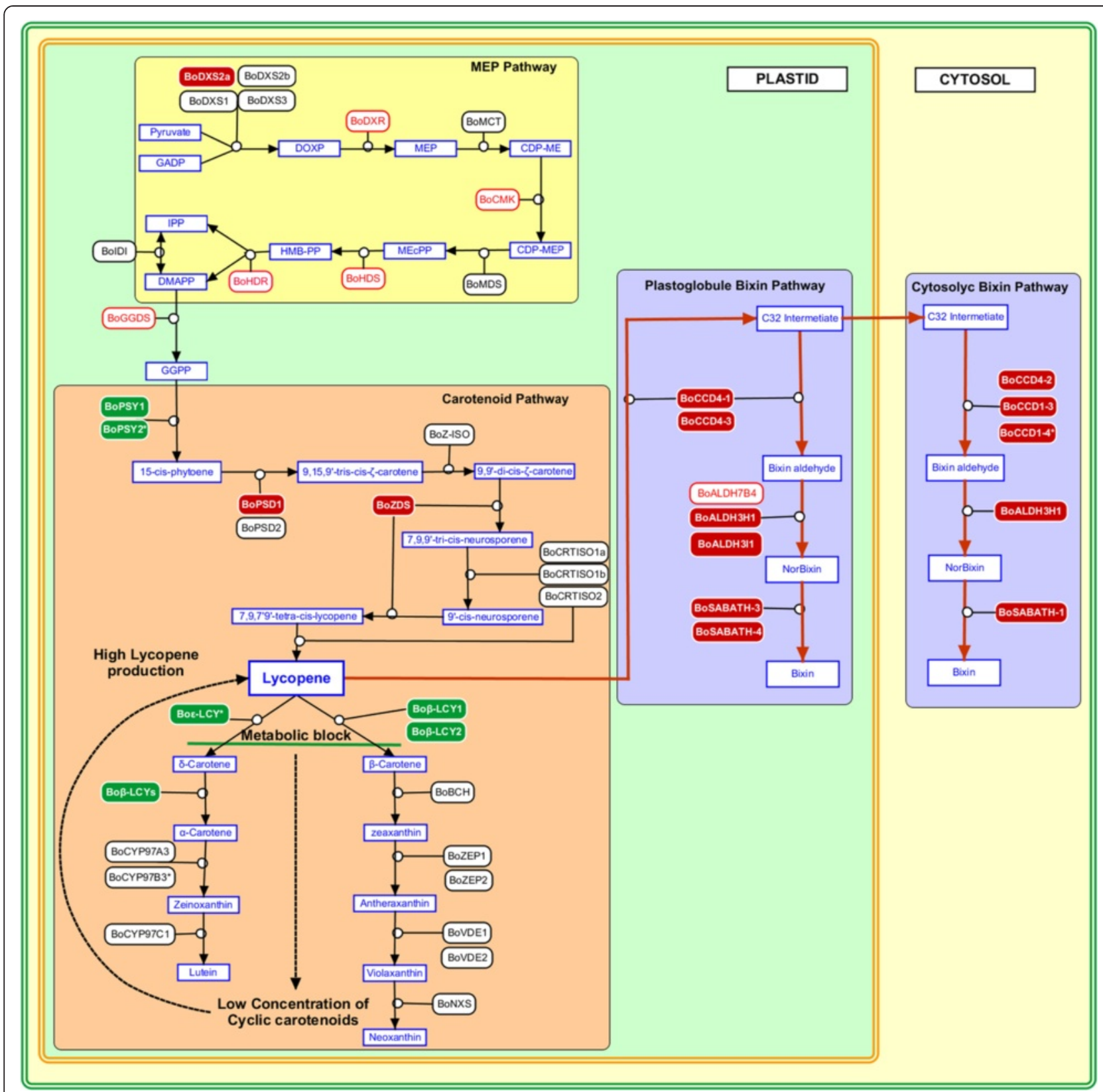

Fig. 7 Model of gene regulation in bixin biosynthesis. Genes with qRT-quantification are represented with filled rectangles. Filled red rectangles indicate genes displaying increased expression in immature seed. Filled green rectangles indicate downregulated genes. Red unfilled rectangles indicate genes represented in the Jako's immature seed library. Asterisks denote partial sequences. The green line indicates blocked downstream process. The green square represents the plastid. The yellow square represents the cytosol. Bright yellow marks the MEP pathway genes. The orange square contains the carotenoid pathway genes and the blue square the bixin pathway. The dashed arrow indicates lycopene feedback regulation. The figure was generated with PathVision 3.1.3 [80]

leading to a decrease in cyclic carotenoids concentration. BoZDS and BoPDS1 overexpression in immature seed (Fig. 6) could thus be a response to low concentrations of end-products in the carotenoid pathway (Fig. 7). Consistent with such a block at the immature seed stage, low $\beta$-carotene and ABA levels [13] correlated with the presence of $P D S$ and absence of lycopene cyclase transcripts $(\beta-L Y C$ and $\varepsilon-L Y C)$ in this tissue [15]. If this block is occurring, the lycopene could accumulate in immature seeds increasing the availability of this compound for the bixin pathway. In conclusion, these results are consistent with the hypothesis that lycopene is the main precursor of bixin [12-14]. 


\section{Identification of new candidates Bixin biosynthesis pathway genes}

Bixin is an orange-red apocarotenoid that accumulates in high quantities in seeds, accounting for $80 \%$ of the total carotenoids. Concentrations of bixin increase continuously during development of immature seeds until they reach maximum size [13]. How is lycopene converted into bixin? The literature indicates the action of three types of enzymes: 1. Carotene cleavage deoxygenase; 2. Aldehyde dehydrogenase; and 3. Methyltransferase. Putative $B$. orellana sequences encoding these enzymes have been described [12]. Surprisingly, we were unable to find transcripts corresponding to the sequences proposed for the above enzymes. Instead, we identified mRNAs encoding different BoCCDs, BoALDH and BoMTs enzymes and believe that these are involved in bixin synthesis. The discrepancy between these and previous findings is explained by the phylogenetic placements of these proteins. The enzymes proposed by Bouvier and co-workers [12] are placed in clades corresponding to monocotyledonous species such as Crocus sativus. Furthermore, BoLCD and BonBMT placement in these clades is well supported with bootstrap values of 99 and $96 \%$, respectively (Additional file 2: Figure S1-S3). It is therefore likely that these cDNAs are not from Bixa orellana, but may have been misplaced in the original study. The sequences proposed here for these enzymes, on the other hand, are in the same phylogenetic branch as cotton, cacao and other dicotyledonous plants and were confirmed as Bixa sequences by PCR amplification using independent Bixa orellana RNA samples.

\section{Carotenoid cleavage dioxygenase candidate proteins in bixin synthesis}

The initial step of bixin synthesis is the $5-6 / 5^{\prime}-6^{\prime}$ oxidative cleavage of lycopene catalyzed by carotenoid cleavage oxygenase to produce bixin aldehyde $[12,14]$. In plants, nine types of carotenoid cleavage dioxygenase have been identified, but only the CCD type 1 and type 4 have been associated with pigment pathways [12, 51-54]. We identified nine putative CCD proteins, four of them CCD type 1 and five type 4 (Table 2 and Additional file 2: Figure S1). As can be seen in Additional file 2: Figure S1, BoCCD1-1 and BoCCD1-2 were closely related to previously isolated CCD1 [13] and they are grouped with monocotyledonous BoCCD1 proteins; this cluster, which was also present in other phylogenetic analysis of CCD family [55], is not well supported with a bootstrap values of 11 in this study and 67 [55], and could be spurious. The gene expression level of previously isolated $B o C C D 1$, correlated with bixin accumulation in B. orellana [13]. This suggests that $B o C C D 1-1$ and $B o C C D 1-2$ could be involved in the cleavage of carotenes to produce seed apocarotenoids, such as ABA and bixin. However, our qRT-PCR analysis indicated that $B o C C D 1-1$ is equally expressed in leaf and immature seed. BoCCD1-2 was preferentially expressed in leaf. Unlike these genes, BoCCD1-3 and BoCCD1-4, were overexpressed 1.5 times and $\sim 10$ times in immature seed compared to leaf, respectively (Fig. 6). This suggests that BoCCD1-3 and $B o C C D 1-4$ are involved in the cleavage of carotenes in immature seed. CCD1 enzymes have the ability, in vitro, to cleave the $5-6 / 5^{\prime}-6^{\prime}$ bond in acyclic carotenoids like lycopene (reviewed in [10]). However, experimental subcellular localizations of CCD1 proteins indicated that they are localized in the cytosol without direct access to lycopene $[54,56]$. In silico prediction of protein properties suggests that BoCCD1-3 is not localized in the chloroplast and presumably does not have direct access to lycopene (Additional file 1: Table S8), therefore it could not be involved in the bixin pathway unless it cleaves lycopene in the cytosol.

CCD4 has the ability to cleave lycopene at the $5,6 / 5^{\prime}, 6^{\prime}$ double bond position and the enzymatic activity is specifically associated with plastoglobules within plastids where it has access to its carotenoid substrates [12, 53, 57-59]. We assembled four cDNAs that were each predicted to encode a complete BoCCD4 ORF (Copy 1-4). The small family formed by these four proteins (Additional file 2: Figure S1) probably originated by duplication, as it appears to be present in other woody plants like $T$. cacao and $P$. trichocarpa. qRT-PCR quantification indicated that BoCCD4-1, BoCCD4-2 and BoCCD4-3 were upregulated in immature seed, suggesting their involvement in the first step of the bixin pathway (Fig. 6). The cDNAs encoding the BoCCD4-2 and BoCCD4-3 proteins were also represented in the previous immature seed library (Table 2) [14]. According to subcellular localization prediction, BoCCD4-1 and BoCCD4-3 are localized in chloroplasts, whereas BoCCD4-2 is localized in the cytosol (Additional file 1: Table S8). Taken together, this evidence suggests that BoCCD4-1 and BoCCD4-3 cleave lycopene in plastids, where bixin is synthesized. We cannot dismiss the possibility that BoCCD1-3 and BoCCD4-2 could participate in the first step of bixin synthesis. Alternatively, the bixin pathway could be localized both in plastids and in the cytosol. In this case, BoCCD4-1 and BoCCD4-3 could cleave one 5-6 lycopene double bound in plastids followed by export of the resulting $\mathrm{C}_{32}$ intermediate to the cytosol. Next, BoCCD1-3 and BoCCD4-2 would cleave the other $5^{\prime}-6^{\prime}$ double bond to produce bixin aldehyde, and cytosolic BoALDHs and BoSABATH would complete the bixin pathway (Fig. 7). The sequential cleavage, first in plastid and then in cytosol, has been demonstrated in the mycorradicin pathway $[60,61]$. 


\section{Aldehyde dehydrogenase candidate proteins in bixin synthesis}

The second step in the bixin pathway is the oxidation of aldehyde groups in bixin aldehyde, into carboxylic acids by aldehyde dehydrogenase [12, 14]. Thirteen distinct families of plant aldehyde dehydrogenases enzymes have been identified, although only ten families (ALDH2, 3, 5, 6, 7, 10, 11, 12, 18 and 22) are present in most plant species [62]. Previously identified $B$. orellana ALDHs that could be involved in the bixin pathway include five clusters of ESTs differentially expressed in immature seed [14], and one BoBADH [GenBank: AJ548846] [12], which appears to be a member of the ALDH2 family, specifically type $2 C_{4}$. BoBADH is related to ALDH2C4 of monocotyledonous plants, especially that of $C$. sativus (Additional file 2: Figure S2). Here, we identified $20 \mathrm{BoALDHs}$ cDNAs from the ten families constituting the common core group (Table 2 and Additional file 2: Figure S2). A partial BoALDH2C4 sequence was also identified in the transcriptome. The fact that ALDH2C4 isolated by Bouvier and co-workers [12] is capable of converting aldehyde groups from bixin aldehyde into carboxylic acids and that it is predicted to localize in the chloroplast (Additional file 1: Table S8), suggests that BoALDH2C4 could catalyze the second step of the bixin pathway in plastids. Alternatively, BoALDH2C4 could be acting in the cytosol because in silico prediction and experimental data indicate that orthologous A. thaliana, G. max, Z. mays, E. parvula and E. salsugineum ALDH2C4 proteins have cytosolic localization [63-66].

Based on subcellular localization prediction, qRT-PCR quantification and presence in the Jako's library [14], the other three BoALDH (BoALDH3H1-1, 3I1, and 7B4) could also be involved in the bixin pathway. The subcellular localization predicted by Plant-mPLoc and PLpred for BoALDH3H1-1, BoALDH3I1 and BoALDH7B4 indicate that they are localized in chloroplast, where they could have access to bixin aldehyde (Additional file 1: Table S8). Additionally, orthologous proteins predicted to be localized in the chloroplast are found in $A$. thaliana, (ALDH3I1), Zea mays (ALDH3H1), E. parvula and E. salsugineum (ALDH3H1 and ALDH3I1), and G. max (ALDH7B4) [64, 65, 67, 68]. BoALDH3H1-1, BoALDH3I1 and $B o A L D H 7 B 4$ are found in the immature seed Jako's library [14]. Moreover, our qRT-PCR analyses indicate that BoALDH3I1 and BoALDH3H1-1 are also upregulated in immature seed (Fig. 6). The subcellular localization of these three proteins in immature seed and the broad range of substrates catalyzed, suggest that these proteins could catalyze the second step in bixin pathway to produce norBixin in plastid or cytosol. The best candidates for this role, however, are BoALDH3I1 and BoADLH3H1 because these enzymes can act on various substrates in plastids
(BoADLH3H1 and BoALDH3I1) or cytosol (BoADLH3H1) (Additional file 1: Table S8) [67]. Moreover, orthologous ALDH3H1 and ALDH3I1 proteins from Synechocystis sp. (SynAdh1), Neurospora crassa (YLO-1) and Fusarium fujikuroi (carD) have the ability to oxidize aldehyde groups from apocarotenoides into carboxylic acids [69-71].

\section{Methyltransferases candidate proteins in bixin synthesis}

The last step of bixin biosynthesis involves a methyltransferase that methylates a norBixin carboxyl group; members of the SABATH methyltransferase family methylate carboxyl groups [23]. This family also includes enzymes that methylate nitrogen atoms. Previous SABATH methyltransferases identified in B. orellana include two clusters of ESTs from the Jako's library [14], and BonBMT, which methylates the carboxyl groups of norBixin (GenBank: AJ548847) [12]. Here, we identified 12 SABATH methyltransferases. None of them is closely related to BonBMT (Additional file 2: Figure S3), which is grouped with the $C$. sativus methyltransferase. BoSAMTH1, 3, 4, 5 and 6 are placed in the same clade, raising the possibility that these proteins share the function of methylating norBixin. In this group of proteins, BoSABATH1 could be involved in bixin synthesis because qRT-PCR indicated that it is overexpressed in immature seed (Fig. 6). Probably, BoSABATH1 methylates norBixin in the cytosol because it is not predicted to have a plastidial localization (Additional file 1: Table S8). qRT-PCR analysis of BoSABATH3 and BoSABATH4 transcripts shows that they are upregulated in immature seed (Fig. 6), thus suggesting that these proteins could be involved in bixin biosynthesis; furthermore, these proteins are represented in the Jako's immature seed library [14]. Subcellular localization prediction indicates that BoSABATH3 and BoSABATH4 are plastidial proteins with direct access to norBixin in chloroplast or chromoplast. Additionally, we identified 26 methyltransferases involved in secondary metabolism (data not shown), but these were not taken into consideration as candidates for norBixin methylation because most methylate oxygen atoms in benzenic rings.

\section{Bixin biosynthesis model}

Bixin production involves the coordinate expression of the MEP, carotenoid and bixin pathways genes in immature seed. Figure 7 illustrates three molecular steps necessary to synthetized bixin: 1 . BoDXS2 $a$ and others MEP genes involved in generation of carotenoids precursor such as $B o D X R$ and $B o H D R$ are induced to produce carotenoids in non photosynthetic tissue. 2. Lycopene cyclase genes $(B o \beta-L Y C 1, B o \beta-L Y C 1$ and $B o \varepsilon-L Y C)$ are turned off, thus blocking metabolic flow toward cyclic carotenoids downstream of lycopene. The low concentrations of $\beta$-carotene 
and xanthophyll, induce the expression of BoPDS1 and $B o Z D S$ and promote lycopene production in plastoglobules of immature seed cells. In this scenario, also PSY should be upregulated, as suggested by its representation in the Jako's library [14]. Surprisingly, the two genes found in this transcriptome were downregulated in our dataset. 3. The BoCCDs (BoCCD1-3, BoCCD1-4, BoCCD4-1, BoCCD4-2 and BoCCD4-3), BoALDH3 (BoALDH3H1 and BoALDH3I1) and BoSABATH (BoSABATH1, BoSABATH3 and BoSABATH4) genes are then turned on leading to lycopene conversion to bixin in plastoglobules or cytosol (Fig. 7).

\section{Conclusion}

Deep sequencing of the Bixa orellana transcriptome enabled the the isolation and characterization of the complete MEP and carotenoid pathway genes. Our inability to find in this transcriptome cDNAs previously identified by Bouvier and co-workers [12], lead us to propose new and alternative enzymes, whose identification was based on the upregulation of the corresponding genes. These findings will help elucidate the regulatory mechanisms controlling the production and accumulation of carotenoid and bixin in B. orellana. For this, characterization of the enzymatic activities proposed here will be necessary. Finally, this information will help identify the candidate genes and mechanisms for variation of apocarotenoids accumulation in achiote varieties, thus facilitating the genetic improvement of achiote for high bixin content.

\section{Methods}

Plant material and total RNA isolation

Samples of young leaves, immature and mature seeds were harvested from $B$. orellana plants cultivated at a commercial plantation in Chicxulub, Yucatán, Mexico. All tissues were obtained from a $B$. orellana accession "Peruana Roja", a variety with pink flowers and high pigment contents characterized by Rivera-Madrid and coworkers [72] (Fig. 1). The fresh tissues were immediately frozen in liquid nitrogen and stored at $-80{ }^{\circ} \mathrm{C}$ until analysis. Total RNA was isolated from leaves, immature and mature seeds from $B$. orellana, accession PR, according to the protocol of Rodríguez-Ávila and co-workers [16].

\section{Illumina sequencing and de novo assembly}

Total RNA from the different tissue was used for the construction of indexed mRNA libraries using KAPA Stranded mRNA-Seq Kit Illumina platform (KAPA Biosystems: KR0960). Libraries were paired end sequenced with 150 cycles in two lanes of the Illumina HiSeq 2500 platform ( $\sim 300$ million reads total) using two insert sizes: 250 bp for read overlap, and $450 \mathrm{bp}$ for paired reads. The long reads are necessary for the assembly of homologous sequences.
Reads were then demultiplexed and preprocessed for quality using scripts developed by the Comai laboratory and available online (http://comailab.genomecenter.ucdavis.edu/ index.php/Barcoded_data_preparation_tools). Reads were trimmed for quality when the average Phred sequence quality over a $5 \mathrm{bp}$ window dropped below 20, trimmed for adapter sequence contamination, and discarded if the final length was shorter than $35 \mathrm{~b}$. For the assembly process reads were processed through the Velvet assembler [17], using kmer sizes ranging from 21 to 53 and a range of expected coverages. The same read set was then also put through CLC Genomics Workbench de novo assembler (http://www.clcbio.com). The Velvet assemblies had duplicates removed and then was combined with the CLC contig set. This combined contig set was reduced to contigs in the size range of $300 \mathrm{bp}-10 \mathrm{kbp}$, and was then put into CAP3 [18] to create transcript contigs. Assemblathon2 Perl script [73] were used to compute assembly statistics. As demonstrated by Ashrafi [19] Velvet and CLC assembly algorithms were found to have complementary qualities for the initial assembly. CAP3 was used as a superassembler to extend Velvet and CLC contigs.

\section{Blast search in public databases}

A local BLAST analysis was performed to compare the achiote transcriptome (52,549 contigs) with three protein databases, NCBI Plant Protein Reference sequence (RefSeq) update in May, 2014, Phytozome v10.0.2 and PLAZA 3.0. The BLASTX algorithm included in bioinformatics package BLAST+ v2.2.29 [74] was used with an e-value cutoff of 1e-6. In order to compare the transcriptome against a previous $B$. orellana EST library [14], a bidirectional BLASTN analysis with e-value cutoff of 1e-100 was performed. The Jako and co-workers EST library is available in NCBI [GenBank: LIBEST_025681 BIXA] [14].

\section{Functional annotation}

For functional annotation, 52,549 contigs were searched against RefSeq using BLASTX algorithm included in bioinformatics package BLAST + v2.2.29. The e-value cutoff of 1e-6 was used for the search and 50 alignments were kept. Gene Ontology terms (GO) from GO database (06/may/14) were extracted from BLASTX results using the BLAST2GO program [75]. To get the functional pathway annotation from KEGG pathways in the curated KEGG GENES database, the KAAS tool (KEGG Automatic Annotation Server) was implemented [76].

\section{Identification of MEP, carotenoid and bixin pathways genes from $B$. orellana transcriptome}

Local TBLASTN with e-value cut off of $1 \mathrm{e}-6$ was performed to search the MEP, carotenoid and bixin pathways genes. Homologous protein from Arabidopsis 
thaliana, Theobroma cacao and Gossypium raimondii were used to make the search against $B$. orellana transcriptome database. If the resultant contigs did not have the complete open reading frame (ORF), then contigs with partial ORFs were isolated and assembled with Lasergen SeqMan software (DNASTAR Inc., Madison, WI, USA).

\section{Phylogenetic analysis}

Phylogenetic reconstruction from proteins codified by a set of 13 single copy genes identified by Duarte and coworkers [21] was based on alignment of concatenated protein sequences from 28 plant species and one moss species. Phylogenetic tree was inferred by the maximumlikelihood method based on Le_Gascuel_2008 (LG) substitution model [77] and Gamma distributed (G). Phylogenetic analysis from $\mathrm{MEP} /$ carotenoid enzymes pathways was based on alignment of concatenated sequences from 29 plant species and one moss species. Phylogenetic tree was inferred by maximum-likelihood method based on Jones-Taylor-Thornton (JTT) substitution model [78] and Gamma distributed with Invariant sites $(\mathrm{G}+\mathrm{I})$. In both cases the analysis were carried out using algorithms included in MEGA6 [79] and the substitution models were predicted by the Best-Fit substitution model (ML) function included in MEGA6. Phylogeny tests were conducted by the bootstrap method (1000 replicates). All positions containing gaps and missing data were eliminated. The alignments of concatenated sequences were performed with the ClustalW algorithm with default parameters on MEGA6. Phylogenetic trees were rooted with Chlamydomonas reinhardtii, a single-cell green alga. Proteins sequences and plant species used are listed in Additional file 1: Table S9.

\section{Gene expression}

The cDNA was synthesized using the SuperScript III First-Strand Synthesis System for the RT-PCR kit (Invitrogen, San Diego, CA) according to the manufacturer's instructions. After reverse transcription, the cDNAs were amplified by qPCR with 40 cycles and with specific primers (Additional file 1: Table S6). A parallel reaction with 40 cycles and specific primers for the $18 S$ rRNA gene (5'-CGGCTACCACATCCAAGGAA-3' and 5'GCTGGAATTACCGCGGCT-3', AF206868) was run as an expression control for each PCR reaction. Three replicates of each PCR reaction were carried out to confirm the results. Gene expression relative to the $18 S$ rRNA gene was assessed using the StepOne Real-Time PCR System (Applied Biosystems catalog number 4376374).

\section{Availability of supporting data}

Supporting data are available in NCBI database.
The Bixa orellana transcriptome has been deposited at Transcriptome Shotgun Assembly project at DDBJ/ EMBL/GenBank under the accession GDKG00000000. The version described in this paper is the first version, GDKG01000000.

BioProject: PRJNA290519 (http://www.ncbi.nlm.nih.gov/bioproject/290519)

BioSample: SAMN03892718 (http://www.ncbi.nlm.nih.gov/biosample/?term=SAMN03892718)

Sequence Read Archive (SRA): SRR2131178 (http:// trace.ncbi.nlm.nih.gov/Traces/sra/

sra.cgi?run=SRR2131178)

\section{Additional files}

Additional file 1: Table S1. BLASTX comparison between the $B$. orellana transcriptome against three databases. Table S2. BLASTN comparison between the $B$. orellana transcriptome and the previous EST library created by Jako and co-workers [GenBank: LIBEST_025681 BIXA]. Table S3. Gene Ontology (GO) annotation. Table S4. Kyoto Encyclopedia of Genes and Genomes (KEGG) annotation. Table S5. Pairwise comparison between amino acid sequences of carotenoid cleavage dioxygenase proteins. Table S6. RT-qPCR primers. Table S7. BLASTx comparison between the $B$. orellana transcriptome and previously identified $B$. orellana proteins .Table S8. Subcellular localization predictions for the BoCCD, BoALDH and BoSABATH proteins. Table S9. Accession number of proteins used in Fig. 1. (ZIP $15364 \mathrm{~kb})$

Additional file 2: Figure S1. Evolutionary relationship of CCDs proteins. Figure S2. Evolutionary relationship of ALDH proteins. Figure S3. Evolutionary relationship of SABATH methyltransferases proteins. Figure S4. Evolutionary relationship of DXS proteins. (ZIP $410 \mathrm{~kb}$ )

\section{Abbreviations}

ABA: Abscisic acid; IPP: Isopentenyl diphosphate; MEP: Methylerythritol phosphate; GGDP: Geranylgeranyl diphosphate; PSY: Phytoene synthase; PDS: Phytoene desaturase; ZDS: Zeta-carotene desaturase; CRTISO: Carotene cis-trans isomerase; Z-ISO: Z-carotene isomerase; $\varepsilon$-LYC: Epsilon-cyclase; $\beta$-LYC: Beta-cyclase; CCD: Carotene cleavage dioxygenase; BoLCD: Lycopene cleavage dioxygenase; BoBALDH: Bixin aldehyde dehydrogenase; BonBMT: Norbixin methyltransferase; DXS: 1-Deoxy-Dxylulose-5-phosphate synthase; DXR: 1-Deoxy-D-xylulose-5-phosphate reductoisomerase; MCT: 2-C-Methyl-D-erythritol 4-phosphate cytidyltransferase; HDS: 4-Hydroxy-3-methylbut-2-en-1-yl diphosphate synthase; GGDS: Geranylgeranyl diphosphate synthase; KEGG: Kyoto encyclopedia of genes and genomes; $\beta C H$ : $\beta$-carotene hydroxylase; CYP97A3: Cytochrome P450-type monooxygenase 97A; CYP97C1: Cytochrome P450-type monooxygenase 97C1; ZEP: Zeaxanthin epoxidase; VDE: Violaxanthin de-epoxidase; IDI: Isopentenyl diphosphate isomerase; CMK: 4-Diphosphocytidyl-2-Cmethyl-D-erythritol kinase; MDS:

2-C-Methyl-D-erythritol 2,4-cyclodiphosphate synthase; HDR: 4-Hydroxy-3methylbut-2-enyl diphosphate reductase; RefSeq: NCBI plant protein reference sequence; GO: Gene ontology terms.

\section{Competing interests}

The authors declare that they have no competing interests.

\section{Authors' contributions}

MAE, VCU and YCC performed experimental molecular biology work. MAE, carried out plants care, providing technical support in the laboratory work at CICY. ML, preparation of raw illumina reads and assembly. YCC carried out the bioinformatics analyses and primers design. RRM, LC conceived, designed and supervised the study. YCC, LC, RRM wrote the manuscript. All authors read and approved the final manuscript. 


\section{Acknowledgments}

This work was supported by the Consejo Nacional de Ciencia y Tecnología (CONACYT) with grant no. 98508, 220259. Yair Cárdenas-Conejo was supported by CONACYT: grant 290754 postdoctoral position. Victor Carballo-Uicab was supported by CONACYT: grant 265369. Work on Bixa orellana in the Comai laboratory was supported by a gift from the Mars Company and by DOE Office of Science, Office of Biological and Environmental Research (BER), grant no. DE-SC0007183 to LC. Authors also thanks Dr. LE. Garza-Caligaris for her comments to the manuscript.

\section{Author details}

${ }^{1}$ Centro de Investigación Científica de Yucatán, A. C. Calle 43 No. 130, Col. Chuburná de Hidalgo, 97200 Mérida, Yucatán, Mexico. ${ }^{2}$ Plant Biology and Genome Center, University of California, Davis, CA 95616, USA.

\section{Received: 12 August 2015 Accepted: 13 October 2015} Published online: 28 October 2015

\section{References}

1. THE ANGIOSPERM PHYLOGENY GROUP. An update of the Angiosperm Phylogeny Group classification for the orders and families of flowering plants: APG III. Bot J Linn Soc. 2009;161:105-21.

2. THE ANGIOSPERM PHYLOGENY GROUP. An update of the Angiosperm Phylogeny Group classification for the orders and families of flowering plants: APG II. Bot J Linn Soc. 2003;141:399-436.

3. DA Dendy $\mathrm{V}$. The assay of annatto preparations by thin-layer chromatography. J Sci Food Agric. 1966;17:75-6.

4. Böhm F, Edge R, Truscott TG. Interactions of dietary carotenoids with singlet oxygen $\left({ }^{1} \mathrm{O}_{2}\right)$ and free radicals: potential effects for human health. Acta Biochim Pol. 2012:59:27-30.

5. Zeevaart JAD, Creelman RA. Metabolism and physiology of abscisic acid. Annu Rev Plant Physiol Plant Mol Biol. 1988;39:439-73.

6. Rohmer M, Seemann M, Horbach S, Bringer-meyer S, Sahm H. Glyceraldehyde 3-phosphate and pyruvate as precursors of isoprenic units in an alternative non-mevalonate pathway for terpenoid biosynthesis. J Am Chem Soc. 1996;118:2564-6.

7. Lichtenthaler HK. The 1-Deoxy-D-Xylulose-5-Phosphate pathway of isoprenoid biosynthesis in plants. Annu Rev Plant Physiol Plant Mol Biol. 1999:50:47-65.

8. Cunningham FX, Gantt E. Genes and enzymes of carotenoid biosynthesis in plants. Annu Rev Plant Physiol Plant Mol Biol. 1998;49:557-83.

9. Nisar N, Li L, Lu S, Khin NC, Pogson BJ. Carotenoid metabolism in plants. Mol Plant. 2015;8:68-82.

10. Walter MH, Strack D. Carotenoids and their cleavage products: biosynthesis and functions. Nat Prod Rep. 2011;28:663-92.

11. Vogel JT, Tan B-C, McCarty DR, Klee HJ. The carotenoid cleavage dioxygenase 1 enzyme has broad substrate specificity, cleaving multiple carotenoids at two different bond positions. J Biol Chem. 2008:283:11364-73.

12. Bouvier F, Dogbo O, Camara B. Biosynthesis of the food and cosmetic plant pigment bixin (annatto). Science. 2003;300:2089-91.

13. Rodríguez-Ávila NL, Narvaez-Zapata JA, Ramirez-Benitez JE, Aguilar-Espinosa ML, Rivera-Madrid R. Identification and expression pattern of a new carotenoid cleavage dioxygenase gene member from Bixa orellana. J Exp Bot. 2011;62:5385-95.

14. Jako C, Coutu C, Roewer I, Reed DW, Pelcher LE, Covello PS. Probing carotenoid biosynthesis in developing seed coats of Bixa orellana (Bixaceae) through expressed sequence tag analysis. Plant Sci. 2002;163:141-5.

15. Rodríguez-Ávila NL, Narvaez-Zapata JA, Aguilar-espinosa ML, Rivera-Madrid R. Regulation of pigment-related genes during flower and fruit development of Bixa orellana. Plant Mol Biol Report. 2011;29:43-50.

16. Rodríguez-Ávila NL, Narvaez-Zapata JA, Aguilar-Espinosa ML, Rivera-Madrid R. Full-length gene enrichment by using an optimized RNA isolation protocol in Bixa orellana recalcitrant tissues. Mol Biotechnol. 2009;42:84-90

17. Zerbino DR, Birney E. Velvet: algorithms for de novo short read assembly using de Bruijn graphs. Genome Res. 2008;18:821-9.

18. Huang X, Madan A. CAP3: a DNA sequence assembly program. Genome Res. 1999;9:868-77.

19. Ashrafi H, Hill T, Stoffel K, Kozik A, Yao J, Chin-Wo SR, et al. De novo assembly of the pepper transcriptome (Capsicum annuum): a benchmark for in silico discovery of SNPS, SSRs and candidate genes. BMC Genomics. 2012;13:571.
20. Wu S, Zhu Z, Fu L, Niu B, Li W. WebMGA: a customizable web server for fast metagenomic sequence analysis. BMC Genomics. 2011;12:444.

21. Duarte JM, Wall PK, Edger PP, Landherr LL, Ma H, Pires JC, et al. Identification of shared single copy nuclear genes in Arabidopsis, Populus, Vitis and Oryza and their phylogenetic utility across various taxonomic levels. BMC Evol Biol. 2010;10:1-18.

22. Logacheva MD, Kasianov AS, Vinogradov DV, Samigullin TH, Gelfand MS, Makeev VJ, et al. De novo sequencing and characterization of flora transcriptome in two species of buckwheat (Fagopyrum). BMC Genomics. 2011;12:1-17.

23. D'Auria JC, Chen F, Pichersky E. The SABATH family of methyltransferases in Arabidopsis thaliana and other plant species. Recent Adv Phytochem. 2003;37:95-125.

24. Rivera-Madrid R, Burnell J, Aguilar-espinosa ML, Rodríguez-Ávila NL, LugoCervantes E, Saenz-Carbonell LA. Control of carotenoid gene expression in Bixa orellana $\mathrm{L}$. leaves treated with norflurazon. Plant Mol Biol Report. 2013;31:1422-32.

25. Soares VLF, Rodrigues SM, de Oliveira TM, de Queiroz TO, Lima LS, HoraJúnior BT, et al. Unraveling new genes associated with seed development and metabolism in Bixa orellana L. by expressed sequence tag (EST) analysis. Mol Biol Rep. 2011:38:1329-40.

26. Hyun TK, Rim Y, Jang H-J, Kim CH, Park J, Kumar R, et al. De novo transcriptome sequencing of Momordica cochinchinensis to identify genes involved in the carotenoid biosynthesis. Plant Mol Biol. 2012;79:413-27.

27. Pan Z, Zeng Y, An J, Ye J, Xu Q, Deng X. An integrative analysis of transcriptome and proteome provides new insights into carotenoid biosynthesis and regulation in sweet orange fruits. J Proteomics. 2012:75:2670-84

28. Grassi S, Piro G, Lee JM, Zheng Y, Fei Z, Dalessandro G, et al. Comparative genomics reveals candidate carotenoid pathway regulators of ripening watermelon fruit. BMC Genomics. 2013;14:781.

29. Motamayor JC, Mockaitis K, Schmutz J, Haiminen N, lii DL, Cornejo O, et al. The genome sequence of the most widely cultivated cacao type and its use to identify candidate genes regulating pod color. Genome Biol. 2013;14:r53.

30. Ming R, Hou S, Feng Y, Yu Q, Dionne-Laporte A, Saw JH, et al. The draft genome of the transgenic tropical fruit tree papaya (Carica papaya Linnaeus). Nature. 2008:452:991-6.

31. Wu GA, Prochnik S, Jenkins J, Salse J, Hellsten U, Murat F, et al. Sequencing of diverse mandarin, pummelo and orange genomes reveals complex history of admixture during citrus domestication. Nat Biotechnol. 2014:32:656-62.

32. Jaillon O, Aury J-M, Noel B, Policriti A, Clepet C, Casagrande A, et al. The grapevine genome sequence suggests ancestral hexaploidization in major angiosperm phyla. Nature. 2007:449:463-7.

33. Wang H, Moore MJ, Soltis PS, Bell CD, Brockington SF, Alexandre R, et al. Rosid radiation and the rapid rise of angiosperm-dominated forests. Proc Natl Acad Sci U S A. 2009;106:3853-8.

34. Qiu Y-L, Li L, Wang B, Xue J-Y, Hendry TA, Li R-Q, et al. Angiosperm phylogeny inferred from sequences of four mitochondrial genes. J Syst Evol. 2010:48:391-425.

35. Morton CM. Newly sequenced nuclear gene $(X d h)$ for inferring angiosperm phylogeny. Ann Missouri Bot Gard. 2011;98:63-89.

36. Fay MF, Bayers C, Alverson WS, Bruijn AY, Chase MW. Plastid rbcL sequence data indicate a close affinity between Diegodendron and Bixa. Taxon. 1998:47:43-50.

37. Alverson WS, Karol KG, Baum DA, Chase MW, Swensen SM, McCourt R, et al. Circumscription of the Malvales and relationships to other Rosidae: evidence from rbcL sequence data. Am J Bot. 1998;85:876-87.

38. Shulaev V, Sargent DJ, Crowhurst RN, Mockler TC, Delcher AL, Jaiswal P, et al. The genome of woodland strawberry (Fragaria vesca). Nat Genet 2011;43:109-16.

39. Zheng C, Sankoff D. Gene order in rosid phylogeny, inferred from pairwise syntenies among extant genomes. BMC Bioinformatics. 2012;13 Suppl 10:S9.

40. Rodríguez-Concepción M. Supply of precursors for carotenoid biosynthesis in plants. Arch Biochem Biophys. 2010;504:118-22.

41. Peng G, Wang C, Song S, Fu X, Azam M, Grierson D, et al. The role of 1deoxy-d-xylulose-5-phosphate synthase and phytoene synthase gene family in citrus carotenoid accumulation. Plant Physiol Biochem. 2013;71:67-76.

42. Ruiz-Sola MÁ, Rodríguez-Concepción M. Carotenoid biosynthesis in Arabidopsis: a colorful pathway. Arabidopsis Book. 2012;10:e0158. 
43. Saladié M, Wright LP, Garcia-Mas J, Rodríguez-Concepción M, Phillips MA The 2-C-methylerythritol 4-phosphate pathway in melon is regulated by specialized isoforms for the first and last steps. J Exp Bot. 2014;65:5077-92.

44. Floss DS, Hause B, Lange PR, Ku H, Strack D, Walter MH. Knock-down of the MEP pathway isogene 1-deoxy-D-xylulose 5-phosphate synthase 2 inhibits formation of arbuscular mycorrhiza-induced apocarotenoids, and abolishes normal expression of mycorrhiza-specific plant marker genes. Plant J. 2008:56:86-100.

45. Bramley PM. Regulation of carotenoid formation during tomato fruit ripening and development. J Exp Bot. 2002;53:2073-87.

46. Giorio G, Stigliani AL, D'Ambrosio C. Phytoene synthase genes in tomato (Solanum lycopersicum L.) - New data on the structures, the deduced amino acid sequences and the expression patterns. FEBS J. 2008;275:527-35.

47. Ronen G, Cohe M, Zamir D, Hirschberg J. Regulation of carotenoid biosynthesis during tomato fruit development: expression of the gene for lycopene epsilon-cyclase is down-regulated during ripening and is elevated in the mutant Delta. Plant J. 1999;17:341-51.

48. Pecker I, Gabbay R, Cunningham FJ, Hirschberg J. Cloning and characterization of the cDNA for lycopene beta-cyclase from tomato reveals decrease in its expression during fruit ripening. Plant Mol Biol. 1996;30:807-19.

49. Simkin AJ, Laboure A-M, Kuntz M, Sandmann G. Comparison of carotenoid content, gene expression and enzyme levels in tomato (Lycopersicon esculentum) leaves. Zeitschrift für Naturforsch C A J Biosci. 2003;58:371-80.

50. Corona V, Aracri B, Kosturkova G, Bartley GE, Pitto L, Giorgetti L, et al. Regulation of a carotenoid biosynthesis gene promoter during plant development. Plant J. 1996;9:505-12.

51. Auldridge ME, McCarty DR, Klee HJ. Plant carotenoid cleavage oxygenases and their apocarotenoid products. Curr Opin Plant Biol. 2006;9:315-21.

52. Rodrigo MJ, Alquézar B, Alós E, Medina V, Carmona L, Bruno M, et al. A novel carotenoid cleavage activity involved in the biosynthesis of Citrus fruit-specific apocarotenoid pigments. J Exp Bot. 2013;64:4461-78.

53. Rubio A, Rambla JL, Santaella M, Gómez MD, Orzaez D, Granell A, et al. Cytosolic and plastoglobule-targeted carotenoid dioxygenases from Crocus sativus are both involved in beta-ionone release. J Biol Chem. 2008;283:24816-25.

54. Frusciante $S$, Diretto G, Bruno M, Ferrante P, Pietrella M, Prado-Cabrero A, et al. Novel carotenoid cleavage dioxygenase catalyzes the first dedicated step in saffron crocin biosynthesis. Proc Natl Acad Sci U S A. 2014;111:12246-51.

55. Priya R, Siva R. Phylogenetic analysis and evolutionary studies of plant carotenoid cleavage dioxygenase gene. Gene. 2014;548:223-33.

56. Auldridge ME, Block A, Vogel JT, Dabney-Smith C, Mila I, Bouzayen M, et al, Characterization of three members of the Arabidopsis carotenoid cleavage dioxygenase family demonstrates the divergent roles of this multifunctional enzyme family. Plant J. 2006;45:982-93.

57. Lashbrooke JG, Young PR, Dockrall SJ, Vasanth K, Vivier MA. Functional characterisation of three members of the Vitis vinifera $\mathrm{L}$. carotenoid cleavage dioxygenase gene family. BMC Plant Biol. 2013;13:156.

58. Ytterberg AJ, Peltier J, Van Wijk KJ. Protein profiling of plastoglobules in chloroplasts and chromoplasts. A surprising site for differential accumulation of metabolic enzymes. Plant Physiol. 2006;140(March):984-97.

59. Vallabhaneni R, Bradbury LMT, Wurtzel ET. The carotenoid dioxygenase gene family in maize, sorghum, and rice. Arch Biochem Biophys. 2010;504:104-11.

60. Floss DS, Walter MH. Role of carotenoid cleavage dioxygenase 1 (CCD1) in apocarotenoid biogenesis revisited. Plant Signal Behav. 2009;4:172-5.

61. Floss DS, Schliemann W, Schmidt J, Strack D, Walter MH. RNA interferencemediated repression of MtCCD1 in mycorrhizal roots of Medicago truncatula causes accumulation of C27 apocarotenoids, shedding light on the functional role of CCD1. Plant Physiol. 2008;148(November):1267-82.

62. Brocker C, Vasiliou M, Carpenter S, Carpenter C, Zhang Y, Wang X, et al. Aldehyde dehydrogenase (ALDH) superfamily in plants: gene nomenclature and comparative genomics. Planta. 2013;237:189-210.

63. Nair RB, Bastress KL, Ruegger MO, Denault JW, Chapple C. The Arabidopsis thaliana REDUCED EPIDERMAL FLUORESCENCE1 gene encodes an aldehyde dehydrogenase involved in ferulic acid and sinapic acid biosynthesis. Plant Cell. 2004;16:544-54.

64. Kotchoni SO, Jimenez-Lopez JC, Kayodé APP, Gachomo EW, Baba-Moussa L. The soybean aldehyde dehydrogenase (ALDH) protein superfamily. Gene. 2012:495:128-33.
65. Hou Q, Bartels D. Comparative study of the aldehyde dehydrogenase (ALDH) gene superfamily in the glycophyte Arabidopsis thaliana and Eutrema halophytes. Ann Bot. 2014;22:1-15.

66. Jimenez-Lopez JC, Gachomo EW, Seufferheld MJ, Kotchoni SO. The maize ALDH protein superfamily: linking structural features to functional specificities. BMC Struct Biol. 2010;10:43.

67. Stiti N, Missihoun TD, Kotchoni SO, Kirch H-H, Bartels D. Aldehyde dehydrogenases in Arabidopsis thaliana: biochemical requirements, metabolic pathways, and functional analysis. Front Plant Sci. 2011;2:1-11.

68. Huang W, Ma X, Wang Q, Gao Y, Xue Y, Niu X, et al. Significant improvement of stress tolerance in tobacco plants by overexpressing a stress-responsive aldehyde dehydrogenase gene from maize (Zea mays). Plant Mol Biol. 2008;68:451-63.

69. Trautmann D, Beyer P, Al-Babili S. The ORF slr0091 of Synechocystis sp. PCC6803 encodes a high-light induced aldehyde dehydrogenase converting apocarotenals and alkanals. FEBS J. 2013;280:3685-96.

70. Estrada AF, Youssar L, Scherzinger D, Al-Babili S, Avalos J. The ylo-1 gene encodes an aldehyde dehydrogenase responsible for the last reaction in the Neurospora carotenoid pathway. Mol Microbiol. 2008;69:1207-20.

71. Díaz-Sánchez V, Estrada AF, Trautmann D, Al-Babili S, Avalos J. The gene carD encodes the aldehyde dehydrogenase responsible for neurosporaxanthin biosynthesis in Fusarium fujikuroi. FEBS J. 2011;278:3164-76.

72. Rivera-Madrid R, Escobedo-GM RM, Balam-Galera E, Vera-Ku M, Harries H. Preliminary studies toward genetic improvement of annatto (Bixa orellana L.). Sci Hortic (Amsterdam). 2006;109:165-72.

73. Bradnam KR, Fass JN, Alexandrov A, Baranay P, Bechner M, Birol I, et al. Assemblathon 2: evaluating de novo methods of genome assembly in three vertebrate species. Gigascience. 2013;2:10.

74. Camacho C, Coulouris G, Avagyan V, Ma N, Papadopoulos J, Bealer K, et al. BLAST+: architecture and applications. BMC Bioinformatics. 2009;10:421.

75. Conesa A, Götz S, García-Gómez JM, Terol J, Talón M, Robles M. Blast2GO: a universal tool for annotation, visualization and analysis in functional genomics research. Bioinformatics. 2005;21:3674-6.

76. Moriya Y, Itoh M, Okuda S, Yoshizagua A, Kanehisa M. KAAS: an automatic genome annotation and pathway reconstruction server. Nucleic Acids Res. 2007;35:182-5.

77. Le SQ, Gascuel O. An improved general amino acid replacement matrix. Mol Biol Evol. 2008;25:1307-20.

78. Jones $D$, Taylor W, Thornton J. The rapid generation of mutation data matrices from protein sequences. Comput Appl Biosci. 1992;8:275-82.

79. Tamura K, Peterson D, Peterson N, Stecher G, Nei M, Kumar S. MEGA5: molecular evolutionary genetics analysis using maximum likelihood, evolutionary distance, and maximum parsimony methods. Mol Biol Evol. 2011;28:2731-9.

80. Kutmon M, van lersel MP, Bohler A, Kelder T, Nunes N, Pico AR, et al. PathVisio 3: an extendable pathway analysis toolbox. PLOS Comput Biol. 2015;11:e1004085.

\section{Submit your next manuscript to BioMed Central and take full advantage of:}

- Convenient online submission

- Thorough peer review

- No space constraints or color figure charges

- Immediate publication on acceptance

- Inclusion in PubMed, CAS, Scopus and Google Scholar

- Research which is freely available for redistribution 